\title{
C-Coupler1: a Chinese community coupler for Earth system modeling
}

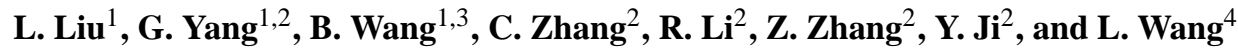 \\ ${ }^{1}$ Ministry of Education Key Laboratory for Earth System Modeling, Center for Earth System Science (CESS), \\ Tsinghua University, Beijing, China \\ ${ }^{2}$ Department of Computer Science and Technology, Tsinghua University, Beijing, China \\ ${ }^{3}$ State Key Laboratory of Numerical Modeling for Atmospheric Sciences and Geophysical Fluid Dynamics (LASG), \\ Institute of Atmospheric Physics, Chinese Academy of Sciences, Beijing, China \\ ${ }^{4}$ College of Global Change and Earth System Science, Beijing Normal University, Beijing, China \\ Correspondence to: L. Liu (liuli-cess@tsinghua.edu.cn), G. Yang (ygw@tsinghua.edu.cn), \\ and B. Wang (wab@tsinghua.edu.cn)
}

Received: 9 May 2014 - Published in Geosci. Model Dev. Discuss.: 11 June 2014

Revised: 23 August 2014 - Accepted: 3 September 2014 - Published: 9 October 2014

\begin{abstract}
A coupler is a fundamental software tool for Earth system modeling. Targeting the requirements of 3-D coupling, high-level sharing, common model software platform and better parallel performance, we started to design and develop a community coupler (C-Coupler) from 2010 in China, and finished the first version (C-Coupler1) recently. C-Coupler1 is a parallel 3-D coupler that achieves the same (bitwise-identical) results with any number of processes. Guided by the general design of C-Coupler, C-Coupler1 enables various component models and various coupled models to be integrated on the same common model software platform to achieve a higher-level sharing, where the component models and the coupler can keep the same code version in various model configurations for simulation. Moreover, it provides the C-Coupler platform, a uniform runtime environment for operating various kinds of model simulations in the same manner. C-Coupler1 is ready for Earth system modeling, and it is publicly available. In China, there are more and more modeling groups using C-Coupler1 for the development and application of models.
\end{abstract}

\section{Introduction}

Climate system models (CSMs) and Earth system models (ESMs) are fundamental tools for the study of global climate change. They play an important role in simulating and understanding the past, present, and future climate. They are always coupled models consisting of several separate interoperable component models to simultaneously simulate the variations of and interactions among the atmosphere, land surface, oceans, sea ice, and other components of the climate system. Following the fast development of science and technology, more and more CSMs, ESMs, and related component models have appeared around the world. For example, more than 50 coupled models participated in the Coupled Model Intercomparison Project Phase 5 (CMIP5), compared to less than 30 coupled models in the previous CMIP3.

A coupler is an important software tool for model development. It links component models together to construct a coupled model, achieves parallel computation among multiple component models, controls the integration of the whole coupled model, and even provides a software platform to allow scientists and engineers to work cooperatively. Most state-of-the-art CSMs and ESMs are constructed with a coupler. With more and more component models (e.g., land ice models, chemistry models, and biogeochemical models) to be added into ESMs, couplers become more and more important for model development. Currently, there are a number of couplers available in the world that have been widely used for model development, e.g., the Ocean Atmosphere Sea Ice Soil coupling software (OASIS) coupler (Redler et al., 2010; Valcke, 2013a), the Model Coupling Toolkit (Larson et al., 2005; Jacob et al., 2005) (MCT), the Earth System Modeling 
Framework (Hill et al., 2004) (ESMF), the Flexible Modeling System (FMS) coupler (Balaji et al., 2006), the CPL6 coupler (Craig et al., 2005) designed for the Community Climate System Model version 3 (Collins et al., 2006) (CCSM3), the CPL7 coupler (Craig et al., 2012) designed for the Community Climate System Model version 4 (Gent et al., 2011) (CCSM4) and the Community Earth System Model (Hurrell et al., 2013) (CESM), and the Bespoke Framework Generator (Ford et al., 2006; Armstrong et al., 2009) (BFG). Most of these couplers provide typical coupling functions (Valcke et al., 2012a), such as transferring coupling fields between component models, interpolating coupling fields between different grids of component models, and coordinating the execution of component models in a coupled model.

Regarding the future development of CSMs and ESMs, we would like to highlight the following ongoing requirements for coupler development:

1. 3-D coupling. Generally, coupling occurs on the common boundaries or domains between component models. In CSMs, most common interfaces between any two component models are on 2-D horizontal surfaces. For example, the common surface between an atmosphere model and an ocean model is on the skin of the ocean, which is also the bottom of the atmosphere. A CSM also needs 3-D coupling - for example, between physics and dynamics in an atmosphere model. In ESMs, component models can share the same 3-D domain. For example, both atmospheric chemistry models and atmosphere models simulate in the 3-D atmosphere space. When coupling them together, especially when their 3D grids are different, 3-D coupling, including transferring and interpolating 3-D coupling fields, needs to be achieved. Some existing couplers, such as the OASIS coupler, MCT, the CPL6 coupler, the CPL7 coupler and ESMF, already provide 3-D coupling functions.

2. High-level sharing. A component model can be shared by different coupled model configurations for various scientific research purposes. In different coupled model configurations, the same component model may have different coupling fields and different common interfaces with other component models. For example, given an atmosphere model, when it is used as a stand-alone component model, there are no coupling fields. When it is used as a component of a CSM, it will provide/obtain 2-D coupling fields for/from other component models. When it is coupled with an atmospheric chemistry model, some 3-D variables with vertical levels become coupling fields. In each coupled model configuration, the atmosphere model can have a branch code version with a specific procedure for providing/obtaining the corresponding coupling fields. When more and more coupled model configurations share the atmosphere model, there will be an increasing number of branch code versions, which will introduce more complexity into the code version control. To facilitate the code version control for sharing a component model, the coupler should enable multiple configurations of coupled models without requiring source code changes to the individual component models and the coupler itself.

3. To develop a model or to achieve a scientific research target, scientists always need to run various kinds of models. For example, to develop an atmosphere model, scientists may want to use various combinations of a single-column model of physical processes, a standalone atmosphere model, an air-sea coupled model, a nested model, a CSM, or an ESM. Moreover, scientists may want to cooperatively use the models from different groups or institutions for a scientific purpose. If the software platforms for these models are not identical, scientists have to invest a lot of effort into learning how to handle model simulations on each platform. To facilitate model development and scientific research, a coupler should be able to integrate various component models and various coupled models on a common model software platform which handles various kinds of model simulations in the same manner, i.e., the same way for creating, configuring, compiling, and running model simulations.

4. Better parallel performance. In the future, the coupler's functions will become more computationally expensive for Earth system modeling in several aspects. First, the coupler will be required to manage the coupling between more and more component models in a coupled model configuration. Second, 3-D coupling will introduce much higher communication and calculation overhead than 2-D coupling. Third, the resolution of component models coupled together will continually increase. Therefore, it will be increasingly important to improve the parallel performance of a coupler.

Motivated by these requirements, in 2010, we started to design and develop a new coupler named "Community Coupler (C-Coupler)", and finished its first version (C-Coupler1) at the end of 2013. C-Coupler1 contains a library with functions for coupling a number of component models together and a uniform runtime environment (we call it the C-Coupler platform) with scripts and configuration files for creating, configuring, compiling, and running model simulations. Besides the typical coupling functions mentioned above, the CCoupler1 libraries achieve parallel 3-D coupling with flexible 3-D interpolation, provide the functionality of integrating external algorithms to enable the same code of C-Coupler and component models shared by various coupled model configurations, and support direct coupling without a specific coupler component to improve the parallel performance. The C-Coupler platform can operate various kinds of model simulations in the same manner. Recently, we successfully used C-Coupler1 to build several model configurations with 
different coupling architectures, and made these model configurations share the same code of the component models and C-Coupler1, as well as the same C-Coupler platform for simulation.

In this paper, we will introduce the general design of CCoupler and show details of C-Coupler1. The remainder of this paper is organized as follows: Sect. 2 briefly introduces existing couplers, Sect. 3 presents the general design of CCoupler, Sect. 4 presents C-Coupler1 in detail, Sect. 5 empirically evaluates C-Coupler 1 , and Sect. 6 discusses the future works for the development of C-Coupler. We conclude this paper in Sect. 7.

\section{Brief introduction to existing couplers}

In this section, we will briefly introduce the OASIS coupler; MCT; ESMF; the FMS, CPL6, and CPL7 couplers; and BFG. More details of these couplers can be found in Valcke et al. (2012a), Redler et al. (2010), Valcke (2013a), Larson et al. (2005), Jacob et al. (2005), Hill et al. (2004), Balaji et al. (2006), Craig et al. (2005, 2012), Ford et al. (2006), and Armstrong et al. (2009).

\subsection{The OASIS coupler}

The European Centre for Research and Advanced Training in Scientific Computing (CERFACS, Toulouse, France) started to develop the OASIS coupler in 1991. OASIS3 (Valcke, 2013a) is a 2-D version of the OASIS coupler. It has been widely used for developing the European CSMs and ESMs. For example, it has been used in different versions of five European CSMs and ESMs that have participated in CMIP5, e.g., CNRM-CM5 (Voldoire et al., 2011), IPSLCM5 (Dufresne et al., 2013), CMCC-ESM (Vichi et al., 2011), EC-Earth V2.3 (Hazelger et al., 2011), and MPIESM (Giorgetta et al., 2013; Jungclaus et al., 2013). OASIS3 uses multiple executables for a coupled model, where OASIS3 itself forms a separate executable for data interpolation tasks and each component model remains a separate executable. It provides an ASCII-formatted "namcouple" configuration file, which is an external configuration file written by users, to specify some characteristics of each coupling exchange, e.g. source component, target component, coupling frequency, and data-remapping algorithm. For data interpolation, OASIS3 can use the remapping weights generated by the 2-D remapping algorithms in the Spherical Coordinate Remapping and Interpolation Package (SCRIP) library (Jones, 1999). The degree of parallelism of OASIS3 is limited to the number of coupling fields, because each process for OASIS3 is responsible for a subset of the coupling fields.

OASIS4 is a 3-D version of the OASIS coupler, which supports both 2-D and 3-D coupling. Similar to OASIS3, OASIS4 also uses multiple executables for a coupled model and can use SCRIP for 2-D interpolation. It also provides a "namcouple" configuration file, and the configuration is described in an XML format. As a 3-D coupler, OASIS4 supports transfer and interpolation for 3-D fields. For 3-D interpolation, OASIS4 itself provides pure 3-D remapping algorithms, e.g., 3-D $n$ neighbor distance-weighted average and trilinear remapping algorithms, and supports 2-D interpolation in the horizontal direction followed by a linear interpolation in the vertical direction. In July 2011, CERFACS stopped the development of OASIS4 and started to develop a new coupler version, OASIS3-MCT (Valcke et al., 2012b; Valcke, 2013b), which further improves the parallelism of OASIS3.

In the following context, we use "namcouple" and "codecouple" to respectively denote the way of specifying characteristics of coupling in configuration files and in source code.

\subsection{The Model Coupling Toolkit}

The Model Coupling Toolkit (MCT) provides the fundamental coupling functions: data transfer and data interpolation, in parallel. MCT represents a coupling field into a 1-D array and uses sparse matrix multiplication to achieve data interpolation. Therefore, it can be used for both 2-D and 3D coupling. For 2-D interpolation, MCT can use remapping weights generated by SCRIP. However, it rarely achieves 3-D interpolation due to the lack of 3-D remapping weights generated by existing remapping software. As a result, MCT is not user-friendly enough in 3-D coupling and users always have to implement 3-D interpolation in the code of component models.

MCT can be viewed as a library for model coupling. It can be directly used to couple fields between two component models where no separate executable is generated for coupling tasks, and can also be used to develop other couplers, e.g., OASIS3-MCT, the CPL6 coupler and the CPL7 coupler.

\subsection{The Earth System Modeling Framework}

The Earth System Modeling Framework (ESMF, www. earthsystemmodeling.org) is a framework for developing models, which consists of a superstructure for creating components and an infrastructure with common coupling functions. A registry of functions in a coupled system was first advanced by the ESMF. A component model can be registered to the ESMF after its routines are organized as standard ESMF methods (initialize, run, and finalize). The ESMF can use both a single executable and multiple executables for a coupled model. It uses "codecouple" configuration for model coupling. For 2-D interpolation, in addition to the typical remapping algorithms such as bilinear and first-order conservative, the ESMF provides a higher-order finite-elementbased patch recovery algorithm to improve the accuracy of interpolation. For 3-D interpolation, similar to the OASIS coupler, the ESMF provides several 3-D remapping algorithms, e.g., trilinear, 3-D $n$ neighbor distance-weighted 
average, and 3-D first-order conservative. For parallelism, the ESMF can remap data fields in parallel and keep bitwiseidentical results when changing the number of processes for interpolation.

In CMIP5, the coupled model NASA GEOS-5 uses ESMF throughout, and the coupled models CCSM4 and CESM1 use the higher-order patch recovery remapping algorithm provided by the ESMF.

\subsection{The FMS coupler}

The Flexible Modeling System (FMS) is a software framework that is mainly developed by and used in the Geophysical Fluid Dynamics Laboratory (GFDL) for the development, simulation and scientific interpretation of atmosphere models, ocean models, CSMs, and ESMs. The coupling between component models in the FMS is achieved by the FMS coupler in parallel. Similar to MCT and ESMF, the FMS coupler uses "codecouple" configuration for model coupling, and can keep bitwise-identical result across different parallel decompositions. One key feature of the FMS coupler is the "exchange grid" (Balaji et al., 2006). Given two component models, the corresponding exchange grid is determined by all vertices in the two grids of these two component models, and the coupling between these two component models is processed on the exchange grid. For example, the coupling fields from the source component model are first interpolated onto the exchange grid and then averaged onto the grid of the target component model. In CMIP5, the CSMs and ESMs (Donner et al., 2011; Dunne et al., 2012) from the GFDL use the FMS as well as its coupler.

\subsection{The CPL6 coupler}

The CPL6 coupler is the sixth version in the coupler family developed at the National Center for Atmospheric Research (NCAR). It is a centralized coupler designed for CCSM3, where the atmosphere model, land surface model, ocean model, and sea ice model are connected to the unique coupler component and the coupling between any two component models is performed by the coupler component. Through integrating MCT, the CPL6 coupler achieves the data transfer and data interpolation in parallel. Moreover, it provides a number of numerical algorithms for calculating and merging some fluxes and state variables for component models. It uses multiple executables for a coupled model, where the coupler component forms a separate executable. Similar to MCT, the CPL6 coupler uses "codecouple" configuration for model coupling. For data interpolation, it always uses the remapping weights generated by SCRIP.

In CMIP5, the CPL6 coupler, as well as the model platform of CCSM3, has been widely used in Chinese coupled model versions, e.g., FGOALS-g2 (Li et al., 2013a), FGOALS-s2 (Bao et al., 2013), BNU-ESM (Ji et al., 2014), BCC-CSM, and FIO-ESM.

\subsection{The CPL7 coupler}

The CPL7 coupler is the latest coupler version from NCAR. It has been used for the CMIP5 models CCSM4 and CESM1. It keeps most of the characteristics from the CPL6 coupler, such as a centralized coupler component, integration of MCT, and flux computation. The most notable advancements from the CPL6 coupler to the CPL7 coupler include the following: (1) the CPL7 coupler does not use multiple executables but single executable for a coupled model and provides a top-level driver to achieve various processor layout and time sequencing of the components in order to improve the overall parallel performance of the coupled model, and (2) a parallel input/output (I/O) library is implemented in the CPL7 coupler to improve the I/O performance.

\subsection{The Bespoke Framework Generator}

The Bespoke Framework Generator (BFG) aims to make the coupling framework more flexible in model composition and deployment. BFG2 is the latest version of BFG. It provides a metadata-driven code generation system where a set of XML-formatted metadata is designed for generating the wrapper code of a coupled model configuration. BFG metadata are classified into three phases: model definition, composition, and deployment. The model definition metadata describe the implementation rules of each component model, including $<$ name $>,<$ type $>,<$ language $>$, $<$ timestep $>$, and $<$ entryPoints $>$. The $<$ entryPoints $>$ consist of a set of entry points each of which corresponds to a user-specified subprogram unit that can be called by the main program in a coupled model. An entry point can contain a number of $<$ data $>$ elements, each of which corresponds to an argument of the entry point. When a $<$ data $>$ element represents an array, the number of dimensions and the bounds for each dimension need to be specified. The composition metadata specify how component models are coupled together with a number of $<$ set $>$ elements. Each $<$ set $>$ element identifies references to a coupling field, where the references are arguments of the corresponding entry points. The deployment metadata specify how the coupled model is mapped onto the available hardware and software resources.

BFG2 designs a "namcouple" approach to separate the code of models from the coupling infrastructure. Although it does not provide coupling functions such as data interpolation, it enables users to choose the underlying coupling functions from other couplers, such as the OASIS coupler. As models are not main programs in a coupled model with BFG2, a single executable or multiple executables can be selected for deploying a coupled model.

\subsection{Summary}

Some existing couplers already support 3-D coupling, such as the OASIS coupler, MCT, ESMF, and the CPL6 and CPL7 
couplers. However, these 3-D coupling functions should be further improved. The 3-D $n$ neighbor distance-weighted average, trilinear, and linear remapping algorithms used in the OASIS coupler and ESMF can result in low accuracy in the interpolation on the vertical direction. MCT, the CPL6 coupler, and the CPL7 coupler generally cannot interpolate in 3$\mathrm{D}$ due to a lack of remapping weights. Moreover, most existing couplers use sparse matrix multiplication to achieve data interpolation. Limited by this implementation, some higherorder remapping algorithms for the 1-D vertical direction, such as spline, cannot be used in 3-D interpolation, because these algorithms cannot be achieved purely by sparse matrix multiplication.

Existing couplers that use "codecouple" configuration, e.g., MCT, ESMF, and the FMS, CPL6, and CPL7 couplers, are not convenient for sharing the same code version of coupler and component models among various coupled model configurations. For example, when increasing coupling fields or component models based on a coupled model configuration, the code of coupler or component models has to be modified. Although some model code could have compiler directives (such as "\#ifdef") for different coupled model configurations, heavy use of compiler directives will make the model code hard to be read and maintain for further development, and the change in coupled model configuration requires a recompiling of model code. Regarding the OASIS coupler, its "namcouple" configuration, which specifies how to transfer and interpolate each coupling field, can decrease code modification requirements when changing the corresponding characteristics of coupling. However, the configuration files in the OASIS coupler do not specify external algorithms for calculating coupling fields, such as flux calculation algorithms. When changing the procedures for calculating coupling fields, the code of component models always has to be modified. Regarding BFG, its metadata can further describe and manage user-specified subprogram units. However, the wrapper code needs to be regenerated and recompiled whenever the metadata changes. Moreover, the description of an array, which specifies the number of dimensions and the bounds for each dimension, is not flexible enough for changing parallel decompositions of models.

There are several model software platforms corresponding to existing couplers which have been successfully used for model development, such as the CCSM3 platform corresponding to the CPL6 coupler, the CCSM4/CESM platform corresponding to the CPL7 coupler, and the FMS. These platforms can configure, compile, and run different kinds of model configurations for simulation. The CCSM4/CESM platform can run stand-alone component models, CSMs, ESMs, etc. However, to make a new standalone component model run on the CCSM4/CESM platform, users have to dramatically modify the code of the component model. For example, when integrating an ocean model version, i.e., MOM4p1 (Griffies et al., 2010), onto the CCSM4/CESM platform for a stand-alone ocean model configuration without coupling with other component models, the code of MOM4p1 needs to be dramatically modified to use the CPL7 coupler as the driver.

Improving parallel performance is always a focus in coupler development. It concerns the parallel performance of both the coupler and the whole coupled model. Similarly, we are concerned with the parallel performance of both the coupler and coupled models in the long-term development of C-Coupler.

\section{General design of C-Coupler}

In this section, we will briefly introduce the general design of C-Coupler. C-Coupler can be viewed as a family of the community coupler developed in China, and C-Coupler1 is the first version following the general design. The future versions of C-Coupler will also follow the general design. In the following context, we first define a general term, experiment model, and then introduce the architecture of the experiment models with C-Coupler and the general software architecture of C-Coupler.

\subsection{A general term for C-Coupler: experiment model}

An experiment model is a model configuration which can run on the C-Coupler platform for simulations. It consists of a certain set of configuration files and a certain set of model code, with a certain set of rules for precompiling. Generally, an experiment model can be any kind of model configuration, such as a single-column model, a stand-alone component model, a regional coupled model, an air-sea coupled model, a nested model, a CSM, an ESM, etc.

\subsection{Architecture of the experiment models with C-Coupler}

To achieve the target of integrating various models on the same common model software platform for a high-level sharing of the component models and for facilitating the construction of a new experiment model, we have designed an architecture for the experiment models with C-Coupler. Figure 1 shows an example of this architecture with a typical CSM, where "ATM", "ICE", "LND", and "OCN" stand for the component models. The key ideas of this design include the following:

1. All experiment models share the same code of CCoupler. Given an experiment model, there could be a separate coupler component which manages the coupling between the component models, while direct coupling without a coupler component is also supported. For example, the red lines in Fig. 1 stand for the direct coupling, where no separate executable is generated for the coupling tasks, and all coupling tasks, such as data 


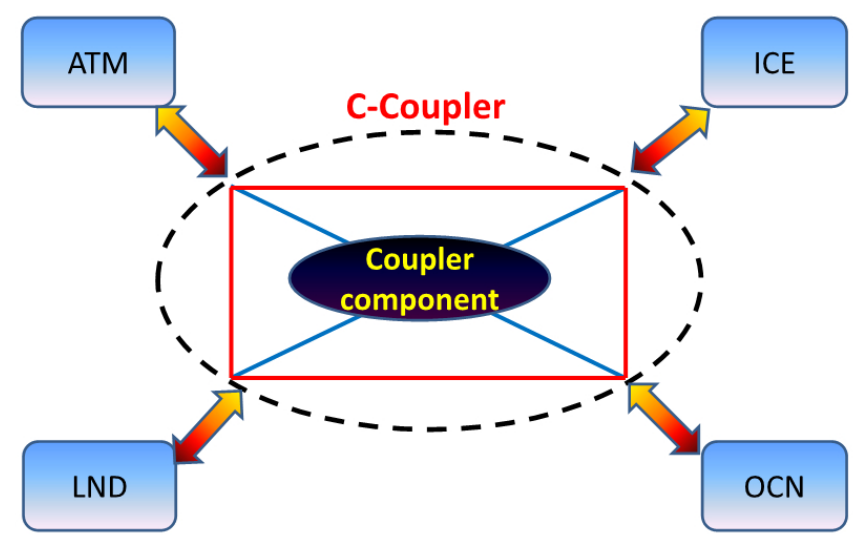

Figure 1. The architecture of the models with C-Coupler.

transfer, data interpolation, and flux computation, are performed through the uniform C-Coupler application programming interfaces (APIs) called by the component models. Compared to the approach with a separate coupler component, direct coupling can reduce the number of data transfers for better parallel performance, but it can lower code modularity. Users can select a separate coupler component, direct coupling, or hybrid for constructing a coupled model configuration. For example, users can use a separate coupler component for a CSM or ESM for better code modularity and use direct coupling for an air-sea coupled model for better parallel performance.

2. When a component model is shared by multiple experiment models, it keeps the same code version in all these experiment models. The code of coupling interfaces in the component model only specifies the input fields that the component model wants and the output fields that the component model can provide, but does not specify how to get the input fields and how to provide the output fields. For example, the source component models, the target component models, and the flux calculation of the coupling fields are not specified in the code of the coupling interfaces.

\subsection{General software architecture of C-Coupler}

Under the guidance of the key ideas, we designed the general software architecture of C-Coupler, which consists of a configuration system, a runtime software system, and a coupling generator, as shown in Fig. 2. In the following context of this subsection, we will further introduce the configuration system and runtime software system. The coupling generator that has not been developed in C-Coupler1 will be further discussed in Sect. 6.

\subsubsection{Configuration system}

In different experiment models, a component model always has different procedures for the input and output fields. For example, given an atmosphere model, in its stand-alone component model configuration, the ocean surface state fields (such as sea surface temperature) are obtained from the I/O data files, while in an air-sea coupled model configuration, the ocean surface state fields are obtained from the ocean model through coupling. Moreover, in these two model configurations, the algorithms for computing the airsea flux (such as evaporation, heat flux, and wind stress) can be different. To make the same code version of a component model shared by various experiment models, C-Coupler should make a procedure adaptively achieve different functions for different model configurations without code modification. We therefore designed a configuration system in C-Coupler. Besides the functionality achieved by the "namcouple" configuration file in the OASIS coupler and BFG, the configuration system of C-Coupler can further specify procedures for coupling. In the following context of this paper, we call such procedures runtime procedures. A runtime procedure consists of a list of algorithms called runtime algorithms. The runtime algorithms can be classified into two categories: internal algorithms and external algorithms. The internal algorithms are implemented within C-Coupler, including the data transfer algorithms, data-remapping algorithms, data I/O algorithms, etc. The external algorithms are always provided by the component models, coupled models, and users. They could be the private algorithms of a component model or common algorithms such as flux calculation algorithms which can be shared by various experiment models.

The configuration system manages the configuration files of software modules and the runtime configuration files of model simulations. The software modules include component models, experiment models, and external algorithms. In detail, the configuration files of a component model specify some characteristics of the component model, e.g., the input and output fields, how to generate the input namelists, and how to compile the code of the model. The configuration files of an experiment model specify how to organize the components, e.g., the components in the experiment model and how each component gets the input fields and provides the output fields. The configuration files of an external algorithm specify the input and output fields of the algorithm. The runtime configuration files specify how to run an experiment model for a simulation, e.g., how to organize the internal algorithms and external algorithms into the runtime procedures for the input and output fields of the components, the coupling frequencies, and the start time and stop time of the simulation.

In detail, the keyword (algorithm name) of each runtime algorithm in a runtime procedure is listed in a simple configuration file. This implementation does not reduce the readability of model code but can make the coupling process 


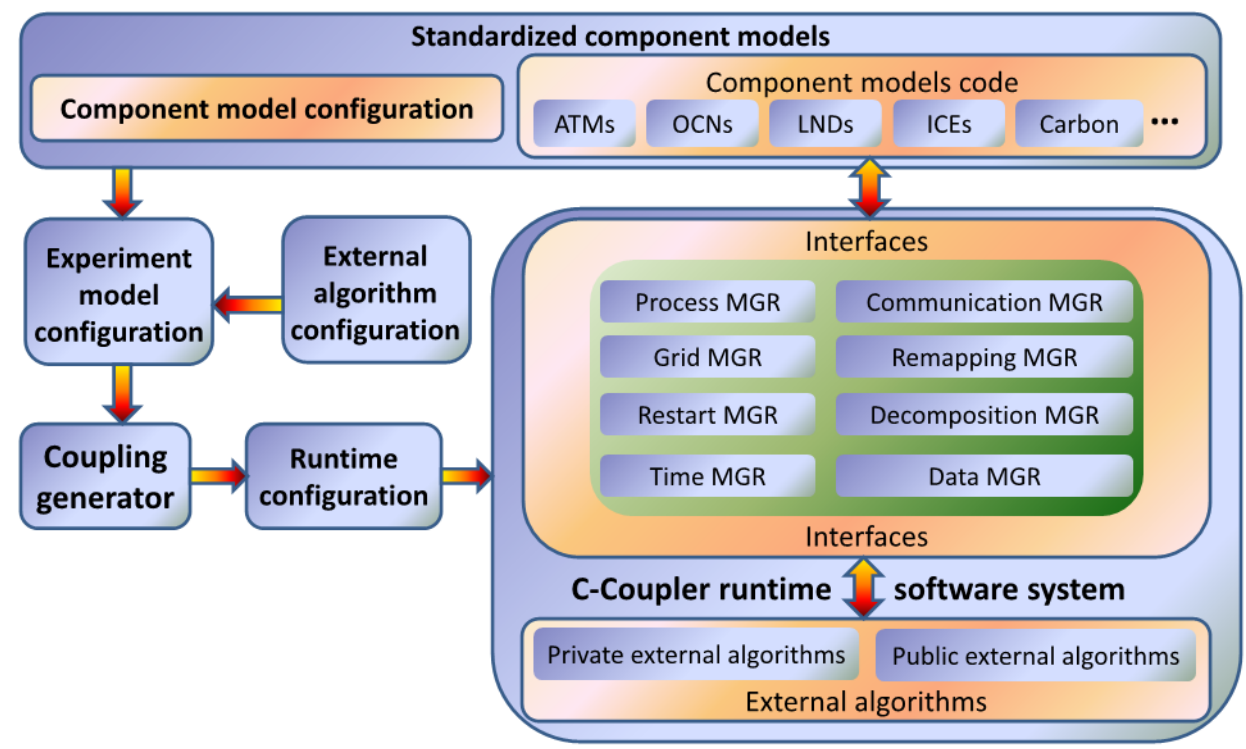

Figure 2. The general software architecture of C-Coupler.

more easily understood. When a runtime procedure is called by model code, the function pointer (some programming languages such as $\mathrm{C}++$ support function pointers) corresponding to each runtime algorithm will be found according to the keyword and then the runtime algorithms can be executed one by one. As the function pointer of a runtime algorithm needs to be searched only one time during the whole simulation, the extra overhead introduced by the approach of the runtime procedure is trivial. The same runtime procedure can achieve different functions in different model configurations through modifying the list of runtime algorithms that is recorded in a configuration file, without modification of model code.

\subsubsection{Runtime software system}

The runtime software system can be viewed as a common, flexible, and extendible library for constructing experiment models and for running model simulations. It enables various kinds of experiment models to share the same code of CCoupler.

First, it provides a set of uniform APIs for integrating component models. With these APIs, a component model can register the model grids, parallel decompositions and I/O fields, and get/put the I/O fields from/into I/O data files or other components, etc.

Second, similar to the ESMF, the runtime software system supports the registry of functions in a coupled system. It provides uniform APIs for integrating external algorithms. A component model can register its private subroutines as external algorithms of C-Coupler. Common algorithms like flux calculation algorithms can also be registered as external algorithms. Therefore, the runtime software system is an extendible library which can integrate more and more common external algorithms, and thus users can have more choices for model simulations. For example, given an air-sea coupled model, if there are several different algorithms for calculating air-sea flux, users can select one of them in a simulation, or two or more of them for sensitivity experiments.

Third, the runtime software system consists of a number of managers (shortened as MGR in Fig. 2), including a communication manager, grid manager, parallel decomposition manager (shortened as decomposition MGR in Fig. 2), remapping manager, timer manager, data manager, restart manager, runtime process manager (shortened as process MGR in Fig. 2), etc. In detail, the communication manager is responsible for allocating and managing the communicators of each component and the whole experiment model. The grid manager manages the grids registered by the component models. The grid can be 1-D, 2-D, 3-D, or even 4-D. The parallel decomposition manager manages the parallel decompositions registered by the component models. The remapping manager manages the remapping algorithms used for coupling. Users can select different remapping algorithms in different simulations of the same experiment model. The timer manager provides timers for triggering the execution of internal and external algorithms. Each algorithm has a timer, and it is executed only when its timer is on. The timer manager can also provide time information for the whole experiment model through the corresponding APIs. The data manager provides uniform APIs for getting the attributes and memory space of fields. A component model can register model fields (including the memory space) as external fields to the data manager. For the internal fields, the data manager will allocate their memory space automatically. The restart manager is responsible for reading fields from the restart I/O data files in a restart run of a model simulation, and writing fields 
into the restart I/O data files when the timer for the restart writing is on. The runtime process manager manages the internal algorithms and the registered external algorithms, organizes these runtime algorithms into a number of runtime procedures, and executes the runtime procedures in a model simulation.

Modularity is an important characteristic of software quality. A coupler is a software tool for improving modularity of coupled models. The software architecture with a set of managers targets better modularity of the runtime software system. It can enhance the independence of each manager, so as to facilitate the advancement of C-Coupler. For example, when one manager is upgraded, the whole C-Coupler is upgraded. Moreover, it can enhance the testing and reliability for each manager. For example, diagnoses can be inserted into the source code of C-Coupler for detecting potential errors in the input and output of a manager.

\section{C-Coupler1: first released version of C-Coupler}

C-Coupler1 is the first version of C-Coupler for public use. Many ideas and concepts from existing couplers have been considered for its design and implementation. As the initial version of C-Coupler, it does not include the coupling generator. However, we carefully developed the configuration system and the runtime software system, which makes C-Coupler1 achieve most of the characteristics in the general design of C-Coupler and reach the target of sharing the same code version of C-Coupler and component models among different experiment models. Moreover, we designed and developed the C-Coupler platform, which enables users to operate various model simulations in the same manner.

C-Coupler1 is a 3-D coupler, where the coupling fields can be $0-\mathrm{D}, 1-\mathrm{D}, 2-\mathrm{D}$, or 3-D. It uses multiple executables for the coupled models, each component of which has a separate executable. It can be used to construct a simple coupler component with a few lines of code, and can also be used for direct coupling between component models without separate executables specifically for the coupling tasks. It does not use the "codecouple" configuration but develops a powerful configuration system. As it is a 3-D coupler, it can interpolate both 2-D and 3-D fields. The runtime software system of C-Coupler1 has been parallelized using the Message Passing Interfaces (MPI) library, while the bitwise-identical result is kept when changing the number of processes for C-Coupler1.

In the following context of this section, we will present the runtime software system, configuration system, and CCoupler platform in C-Coupler1, and then introduce how to couple a component model and the enhancement for reliability of software.

\subsection{The runtime software system}

The runtime software system is a parallel software library programmed mainly in $\mathrm{C}++$ for better code modularity. It provides APIs mainly in Fortran, because most component models for Earth system modeling are programmed in Fortran. In the following context, we will introduce the technical features of the runtime software system, including the APIs, each manager, and parallelization.

\subsubsection{The APIs}

Table 1 lists out the APIs provided by C-Coupler1, which can be classified into four categories: the main driver, registration, restart function, and time information. Besides the brief description of each API in Table 1, we would like to further introduce two APIs: $c_{-}$coupler_execute_procedure and c_coupler_register_model_algorithm.

The API c_coupler_execute_procedure takes the name of a runtime procedure as an input parameter, while the algorithm list for the runtime procedure is specified in the corresponding runtime configuration files. Thus, a runtime procedure can keep the same name in various experiment models, and users can make the same runtime procedure perform different tasks through modifying the runtime configuration files that can be viewed as a part of input of a model simulation. As a result, a component model can keep the same code version in various experiment models sharing it.

Almost all APIs are in Fortran except the $\quad$ __coupler_register_model_algorithm. The c_coupler_register_model_algorithm is in $\mathrm{C}++$ because most Fortran versions do not support function pointer. A private external algorithm (or subroutine) registered by a component model through this API does not have explicit input and output fields. The input and output of such a algorithm are specified implicitly in the code (always Fortran code) through use of public variables of component models. In the future version of C-Coupler, we may enable the private external algorithms to have explicit input and output fields.

\subsubsection{The implementation of managers}

\section{The communication manager}

The communication manager adaptively allocates and manages the MPI communicators for the MPI communications within and between the components of an experiment model. It also provides some utilities for other managers, such as getting the ID of a process in the communicator of a component or in the global communicator.

\section{The grid manager}

The grid manager utilizes a multidimensional remapping software CoR (Common Remapping) (Liu et al., 2013a, 
Table 1. The APIs provided by C-Coupler1.

\begin{tabular}{|c|c|c|}
\hline Classification & API & Brief description \\
\hline \multirow[t]{3}{*}{ Main driver } & c_coupler_initialize & $\begin{array}{l}\text { This API initializes the runtime software system. A component model } \\
\text { can obtain its MPI communicator with this interface. }\end{array}$ \\
\hline & c_coupler_finalize & This API finalizes the runtime software system. \\
\hline & c_coupler_execute_procedure & $\begin{array}{l}\text { This API invokes the runtime software system to run the correspond- } \\
\text { ing runtime procedure which consists of a list of runtime algorithms } \\
\text { specified by the corresponding runtime configuration files. The cor- } \\
\text { responding runtime procedure could be empty without any runtime } \\
\text { algorithms. A component model can have multiple different runtime } \\
\text { procedures. }\end{array}$ \\
\hline \multirow[t]{4}{*}{ Registration } & c_coupler_register_model_data & $\begin{array}{l}\text { This API registers a field of component model to enable the runtime } \\
\text { software system to access the memory space of this field. }\end{array}$ \\
\hline & c_coupler_withdraw_model_data & $\begin{array}{l}\text { This API withdraws a field of component model from the runtime } \\
\text { software system which has been registered before. }\end{array}$ \\
\hline & c_coupler_register_decomposition & $\begin{array}{l}\text { This API registers a parallel decomposition to the runtime software } \\
\text { system. A component model can register multiple different parallel } \\
\text { decompositions, even on the same horizontal grid. }\end{array}$ \\
\hline & c_coupler_register_model_algorithm & $\begin{array}{l}\text { This API registers an algorithm (also known as a subroutine) of a com- } \\
\text { ponent model as an external algorithm of the runtime software system. }\end{array}$ \\
\hline \multirow[t]{2}{*}{ Restart function } & c_coupler_do_restart_read & $\begin{array}{l}\text { This API reads in the data value of fields in a restart run of a model } \\
\text { simulation. }\end{array}$ \\
\hline & c_coupler_do_restart_write & This API writes out the data value of fields for restart run. \\
\hline \multirow[t]{20}{*}{ Time information } & c_coupler_get_current_calendar_time & This API gets the calendar time of the current step. \\
\hline & c_coupler_get_nstep & $\begin{array}{l}\text { This API gets the number of the current step from the start of the } \\
\text { model simulation. }\end{array}$ \\
\hline & c_coupler_get_num_total_step & This API gets the number of total steps of the model simulation. \\
\hline & c_coupler_get_step_size & This API gets the number of seconds of the time step. \\
\hline & c_coupler_is_first_restart_step & $\begin{array}{l}\text { This API checks whether the current step is the first step of a restart } \\
\text { run. }\end{array}$ \\
\hline & c_coupler_is_first_step & $\begin{array}{l}\text { This API checks whether the current step is the first step of an initial } \\
\text { run, which also means whether the number of the current step is } 0 \text {. }\end{array}$ \\
\hline & c_coupler_advance_timer & This API advances the time of simulation. \\
\hline & c_coupler_check_coupled_run_finished & This API checks whether the model simulation ends. \\
\hline & c_coupler_check_coupled_run_restart_time & $\begin{array}{l}\text { This API checks whether the current step is time for writing fields into } \\
\text { I/O data files for restart run. }\end{array}$ \\
\hline & c_coupler_get_current_num_days_in_year & $\begin{array}{l}\text { This API gets the number of days elapsed since the first day of the } \\
\text { current year. }\end{array}$ \\
\hline & c_coupler_get_current_year & This API gets the year of the current step. \\
\hline & c_coupler_get_current_date & This API gets the date of the current step. \\
\hline & c_coupler_get_current_second & This API gets the second of the current step. \\
\hline & c_coupler_get_start_time & This API gets the start time of the model simulation \\
\hline & c_coupler_get_stop_time & This API gets the end time of the model simulation. \\
\hline & c_coupler_get_previous_time & This API gets the time of the previous step. \\
\hline & c_coupler_get_current_time & This API gets the time of the current step. \\
\hline & c_coupler_get_num_elapsed_days_from_start & $\begin{array}{l}\text { This API gets the number of days elapsed since the start time of the } \\
\text { model simulation. }\end{array}$ \\
\hline & c_coupler_is_end_current_day & $\begin{array}{l}\text { This API checks whether the current step is the last step of the current } \\
\text { day. }\end{array}$ \\
\hline & c_coupler_is_end_current_month & $\begin{array}{l}\text { This API checks whether the current step is the last step of the current } \\
\text { month. }\end{array}$ \\
\hline
\end{tabular}


b; it can be downloaded through "svn-username=guestpassword=guest co http://thucpl1.3322.org/svn/coupler/ CoR1.0") to manage the grids with dimensions from 1-D to 4-D. In a model simulation, the grids of component models are registered to the grid manager with a script of CoR, where the grid data (such the latitude, longitude and mask corresponding to each grid cell) are always read from I/O data files. Besides the support of multiple dimensions of grids, another advantage of using CoR for grid management is that it can detect the relationship between to two grids; for example, a 2-D grid is the horizontal grid of a 3-D grid with vertical levels. Moreover, for horizontal 2-D grid, CoR can support logically rectangular and unstructured grids, such as a cubic spherical grid.

\section{The parallel decomposition manager}

Most of component models for Earth system modeling have been parallelized using the MPI library, where the whole domain of each component model, which always is a 3-D grid with vertical levels, is decomposed into a number of subdomains for parallelization and each process of the component model is responsible for a subdomain. We refer to the decomposition from the whole domain into subdomains as parallel decomposition. In C-Coupler1, each parallel decomposition managed by the parallel decomposition manager is based on a 2-D horizontal grid that has been registered to the grid manager; the parallel decomposition on the vertical subgrid of the 3-D grid is not yet supported. To register a parallel decomposition, C-Coupler1 uses an implementation derived from MCT: each process of a component model enumerates the global index (the unique index in the whole domain) of each local cell (each cell in the subdomain of this process) in the corresponding horizontal grid. A component model can register multiple parallel decompositions on the same horizontal grid, while each parallel decomposition has a unique name which is treated as the keyword of it.

\section{The remapping manager}

The remapping manager utilizes CoR to achieve the data interpolation function. There are several remarkable advantages of using CoR for interpolation. First, it can help the Coupler1 to remap the field data on 1-D, 2-D, and 3-D grids. Second, it can generate remapping weights using its internal remapping algorithms and can also use the remapping weights generated by other software, such as SCRIP. Third, it is designed to be able to interpolate field data between two grids with any structures, which makes C-Coupler1 able to be used more extensively. Similar to other couplers such as the OASIS coupler, MCT, ESMF, and the CPL6/CPL7 coupler, C-Coupler1 can utilize the remapping weights generated offline by remapping software such as CoR and SCRIP. The $\mathrm{I} / \mathrm{O}$ data files for the offline remapping weights are specified in a script of CoR, the same script for registering the grids.
In different simulations of the same experiment model, users can select different remapping algorithms through modifying the script.

Considering that the vertical grids (such as a sigma-p grid) of component models may be changed during the model execution, the 1-D remapping weights for the "2-D + 1-D" interpolation can be generated online in parallel by $\mathrm{C}$-Coupler1 in the execution of a model simulation. This online weight generation can scale well because the vertical grid is not decomposed for parallelization and each process can generate the 1-D remapping weights independently.

CoR makes C-Coupler1 more flexible in 3-D interpolation when compared with existing couplers. First, CoR supports the "2-D + 1-D" approach to interpolate data between two 3 -D grids, where a 2-D remapping algorithm is used for the interpolation between the 2-D horizontal subgrids and a 1$D$ remapping algorithm is used for the interpolation between the 1-D vertical subgrids. Given that there are $M$ 2-D remapping algorithms and $N$ 1-D remapping algorithms, there are $M \times N$ selections for 3-D interpolation. Second, CoR supports both sparse matrix multiplication and equation group solving for interpolation calculation. Thus, it can provide some higher-order remapping algorithms, such as spline, that require a tri-diagonal equation group to be solved. Moreover, it makes C-Coupler1 able to handle some unstructured grids. For example, when the source grid is a cubic spherical grid, the bilinear remapping algorithm in SCRIP cannot be used, while CoR can handle this case.

At the present time, there are several remapping algorithms available in CoR. For the 2-D horizontal interpolation, CoR provides three remapping algorithms: first-order conservative, bilinear, and 2-D $n$ neighbor distance-weighted average. For the 1-D vertical interpolation, CoR provides two remapping algorithms: linear and spline. Currently, there are no pure 3-D remapping algorithms implemented, and 3-D interpolation is achieved by the "2-D + 1-D" approach. In the detailed implementation for this approach, the 2-D and 1-D remapping weights are managed separately, and there could be a number of matrixes for 2-D remapping weights and for 1-D remapping weights. For some cases, the matrixes for the 2-D and 1-D remapping weights can be merged into one large matrix of 3-D remapping weights. We do not select this implementation, because it can dramatically increase the calculation for 3-D interpolation. Moreover, this implementation cannot handle the vertical spline interpolation, which requires equation group solving. For each spline interpolation on the vertical direction, the coefficient matrix of the equations can be pre-calculated by $\mathrm{CoR}$ when generating the offline remapping weights. During the model execution, CCoupler1 will call the CoR functions to solve the equation group. The vertical interpolation scales well because the vertical grid is not decomposed for parallelization and each process can handle the vertical interpolation independently. 


\section{The timer manager}

The timer manager coordinates the component models in a coupled model to advance the simulation time in an orderly. In the runtime software system, each coupling field can have a set of timers for periodically triggering the operations on it. For example, a coupling field always has a timer for data transfer and a timer for data interpolation. Moreover, each external algorithm has a timer to periodically trigger its execution. There are three elements in a timer: the unit of frequency, the count of frequency, and the count of delay. The unit and the count of frequency specify the period of the timer. The count of delay specifies a lag of the time during which the corresponding operation or algorithm will not be executed. The unit of frequency can be "years", "months", "days", "seconds", and "steps", where "steps" means the time step of calling the API c_coupler_advance_timer. For example, the timer $<10$, steps, $15>$ means that the corresponding operation or algorithm will be executed at the steps with number $10 \times N+15$, where $N$ is a nonnegative integer. Sequential and concurrent runs between component models can be achieved through cooperatively setting the delay of the timers.

Besides managing all the timers, the timer manager provides interfaces for getting the information of model time (e.g., calendars) during a simulation.

\section{The data manager}

The fields managed by the data manager include the external fields which are registered by the components with APIs and the internal fields which are automatically allocated by the data manager. A coupling field, such as sea surface temperature (SST), can have different instances in a coupled model configuration due to different parallel decompositions and different grids. There is a keyword for each field instance, which consists of the name of the field, the name of the corresponding component model, the name of the corresponding parallel decomposition, and the name of the corresponding grid. To define a field instance, the corresponding four names must have been defined or registered to the runtime software system. For an external field instance, these four names are specified when a component model registers this field instance through calling the corresponding API, while for an internal field instance, these four names are specified in configuration files. For the scalar field which is not on a grid, the corresponding parallel decomposition and grid are marked as "NULL".

All instances of a coupling field share the same field name. The field values in one instance can be transformed into another instance through data transferring between two component models and data interpolation within a component model. All component models in a coupled model share the names of the coupling fields. All legal field names are listed in the configuration files, with other attributes such as the long name (also known as the description of the field) and the unit.

The data manager achieves several advantages beyond existing solutions. First, a 2-D field and a 3-D field can share the same 2-D parallel decomposition, while their corresponding grids are different, where the 2-D grid corresponding to the 2-D field is a subgrid of the 3-D grid corresponding to the 3-D field. Second, the data manager unifies the management of different kinds of field instances, such as with different grids, different parallel decompositions, and different data types (i.e., integer and floating point). As a result, the data transfer algorithm can transfer different kinds of field instances at the same time for better communication performance. Third, the data manager helps improve the reliability for model coupling. For example, the remapping manager can examine whether the grids of source fields and target fields match the grids of the remapping weights.

\section{The restart manager}

For reading/writing fields from/into the restart I/O data files, the restart manager iterates on each field managed by the data manager. For the internal fields, the restart manager can automatically detect the fields which are necessary for restarting the model simulation. For an external field, the corresponding component can specify whether this field is necessary for restarting when registering it with the C-Coupler API. As a result, a component model has more selections for achieving the restart function. It can still use its own restart system or register all fields for restart as external fields to the data manager.

\section{The runtime process manager}

The runtime process manager is responsible for running the list of runtime algorithms in each runtime procedure during a model simulation. Besides the external algorithms, including the private algorithms registered by the component models and the common flux calculation algorithms, there are several algorithms internally implemented in the runtime software system, e.g., the data transfer algorithm, data-remapping algorithm, and data $\mathrm{I} / \mathrm{O}$ algorithm. The data transfer algorithm is responsible for transferring a number of fields from one component to another. The fields transferred by the same data transfer algorithm can have different number of dimensions, different data types, different parallel decompositions, different grids, different frequency of transfer, etc. The data transfer algorithm packs all fields that are to be transferred at the current time step into one package to improve the communication performance.

The data-remapping algorithm uses the corresponding algorithm in CoR as a kernel for implementation. It can remap several fields at the same time for better parallel performance. Multiple fields in a data-remapping algorithm share the same parallel decomposition, the same grid, and the same 
timer, while the data types (i.e., single-precision and doubleprecision floating point) can be different.

The data I/O algorithm currently utilizes the serial I/O to read/write multiple fields which are managed by the data manager from/into the data I/O files. The multiple fields in a data $\mathrm{I} / \mathrm{O}$ algorithm share the same timer, but they can have different parallel decompositions, different grids, and different data types. The fields of a data I/O algorithm are specified in the corresponding runtime configuration file. For the future version of C-Coupler, we will further improve the I/O performance with parallel I/O for higher-resolution models.

\subsubsection{Parallelization}

As previously mentioned, the runtime software system has been parallelized using the MPI library and achieves bitwiseidentical results when changing the number of processes. Here we would like to further introduce some details, including the parallelization of the data transfer algorithm, the parallelization of the data-remapping algorithm, and the default parallel decomposition.

\section{Parallelization of the data transfer algorithm}

This parallelization is derived from existing couplers such as MCT. For a coupling field instance transferred by the data transfer algorithm, it has a parallel decomposition in the source component and another parallel decomposition in the target component, and these two parallel decompositions share the same horizontal grid. A process in the source component will transfer the data of this field to a process in the target component only when the corresponding subdomains on these two processes have common cells. As this implementation does not involve collective communications and there are always multiple processes to execute the source component and the target component, the data transfer algorithm can transfer the coupling fields in parallel.

\section{Parallelization of the data-remapping algorithm}

The data-remapping algorithm interpolates a number of fields from the source grid to the target grid. To cause the fields to be interpolated in parallel, C-Coupler1 uses an approach from MCT, which generates an internal parallel decomposition for rearranging coupling fields before interpolating. The internal parallel decomposition is on the source grid and determined by the remapping weights and the parallel decomposition corresponding to the target grid. For example, given that global cell $y$ of the target grid is assigned to process $p$, and given a remapping weight $\langle x, y, w\rangle$, where $x$ specifies a global cell in the source grid and $w$ is a weight value, the internal parallel decomposition for process $p$ will include the global cell $x$ of the source grid. After rearranging the fields according to the internal parallel decomposition using the data transfer algorithm, process $p$ can interpolate the fields locally. This implementation avoids the reduction for sum between multiple processes of a component model so as to make the data-remapping algorithm achieve bitwiseidentical results when using different numbers of processes. Although it can increase the overhead when rearranging coupling fields, the collective communication for the reduction for sum between multiple processes can be avoided.

\section{Default parallel decomposition}

In an experiment model, not all parallel decompositions are specified by the component models through registration. For example, the parallel decompositions in a coupler component are not determined by any component model. Therefore, the runtime software system provides a default parallel decomposition. Given the number of processes $N$, the default parallel decomposition partitions a horizontal grid into $N$ distinct subdomains without common cells, and the number of cells in each subdomain is around the average number.

\subsection{Configuration system}

As we did not develop the coupling generator in C-Coupler1, we did not develop the configuration files of experiment models accordingly. In the following context of this subsection, we will introduce the configuration files of the component models, the configuration files of the external algorithms, and the runtime configuration files of the model simulations.

\subsubsection{The configuration files of the component models}

Each component model has a set of configuration files which specify the following information:

1. How to generate input namelist files. The generation of input namelist files is specified in a script named config.sh. When users configure a model simulation, config.sh will be invoked to generate the namelist files.

2. Where the source code is. The locations of source code are specified in a script named form_src.sh. A location can be a specific code file or a directory, which means that all code files under it need to be compiled. When users compile the model code for a simulation, form_src.sh will be invoked.

3. How to compile the model code. The compilation of a model code is specified in a script named build.sh. It enhances flexibility for compilation: a component model can use the compilation utility provided by the C-Coupler platform or use its own compilation system. When users compile the model code for a simulation, build.sh will be invoked.

4. Compiling options for the component model, which are specified in a configuration file named compiler.cfg. 


$\begin{array}{lll}\text { itime } & \text { NULL } & \text { NULL } \\ \text { tbot } & \text { atm_2D_decomp1 } & \text { atm_2D_grid1 } \\ \text { tbot } & \text { atm_2D_decomp2 } & \text { atm_2D_grid1 } \\ \text { tbot } & \text { atm_2D_decomp3 } & \text { atm_2D_grid2 } \\ \text { tbot } & \text { atm_2D_decomp4 } & \text { atm_2D_grid2 } \\ \text { qpert } & \text { atm_2D_decomp1 } & \text { atm_3D_grid1 } \\ \text { qpert } & \text { atm_2D_decomp3 } & \text { atm_3D_grid2 }\end{array}$

Figure 3. A configuration file for an atmospherical model to register field instances to C-Coupler.

5. The information of the field instances that will be registered to C-Coupler1. Figure 3 shows an example for the corresponding configuration file, where each line corresponds to a field instance. The first column specifies the field names, the second column specifies the parallel decompositions, and the last column specifies the grids. A component model can register multiple instances for the same field, on different grids or different parallel decomposition. From Fig. 3, we can find that $a t m \_2 D \_g r i d l$ is a subgrid of $a t m \_3 D \_g r i d l$ because they can both share the same parallel decomposition, atm_2D_decomp1. Similarly, atm_2D_grid2 is a subgrid of atm_3D_grid2. This configuration file will be queried when the atmosphere model registers a field instance.

\subsubsection{The configuration files of the external algorithms}

As shown in Fig. 4, an external algorithm has a configuration file for the main information. In the main information (Fig. 4a), the first line is the algorithm name for searching the corresponding function pointer, and the second line specifies a timer for triggering the execution of the external algorithm. The last two lines specify the name of two configuration files for the input and output field instances, respectively. For the private external algorithm that does not have input and output fields, these two lines are set to "NULL". For a field instance that is both an input and output of the external algorithm, it should be referenced in the two configurations files. Figure $4 \mathrm{~b}$ shows an example for how to describe the input (or output) field instances, where each line corresponds to a field instance. Columns 1-4 specify the keyword for each field instance, while the last column specifies the data type.

\subsubsection{The runtime configuration files of the model simulations}

Corresponding to the design and implementation of the runtime software system, the runtime configuration files contain the following information about a model simulation: (1) the configuration files of the corresponding components and external algorithms; (2) a CoR script to specify the grids and the weights for remapping algorithms; (3) the configuration files for each runtime procedure in each component that will be further illustrated in Sect. 5.1; and (4) the namelist of the model simulation that is common to all components, including the start time, stop time, run type (initial run or restart run), etc.

\subsubsection{Summary}

Similar to the OASIS coupler and BFG, C-Coupler develops a "namcouple" configuration system for specifying the coupling characteristics of an experiment model. BFG defines the metadata of coupling characteristics in three phases: model definition, composition, and deployment. Regarding C-Coupler, the configuration files of a component model function similarly to the model definition metadata and the configuration files of an experiment model function similarly to metadata composition. Generally, the "namcouple" implementation in C-Coupler is different from that in the OASIS coupler and BFG as follows:

1. The procedure registration system with runtime procedures and runtime algorithms can support a wide number of coupled model configurations while maintaining the same codebase for component models.

2. Grids and parallel decompositions are referenced by name in the configuration system. As a result, most of the configuration files of an experiment model can remain the same when changing the parallel settings, model resolutions, or model grids.

3. With the help of CoR, the configuration system can bridge the relationship between parallel decompositions, grids, remap weights, etc. Therefore more kinds of diagnoses can be conducted to make C-Coupler and experiment models more reliable. For example, the remapping manager can examine whether the grids of source fields and target fields match the grids of remapping weights.

\subsection{The C-Coupler platform}

To facilitate operating the simulation of various experiment models with C-Coupler1, we designed and developed the CCoupler platform. Figure 5 shows its general architecture. It manages the input data and the software modules for the experiment models, including standardized component models, external algorithms, runtime configuration files of the model simulations, and the runtime software system. There are four steps to operating a model simulation on the C-Coupler platform: "create case", "configure", "compile", and "run case". The "create case" step means creating a model simulation. There are two approaches for creating a model simulation: creating a default simulation of an experiment model using the script "create_newcase" and creating a model simulation from an existing model simulation. The C-Coupler platform 


$$
\begin{aligned}
& \text { fields_mult } \\
& \text { seconds } \quad 3600 \\
& \text { fields_mult_input_fields.cfg } \\
& \text { fields_mult_output_fields.cfg }
\end{aligned}
$$

(a) Main information for the external algorithm

$\begin{array}{lllll}\text { itime } & \text { atm_model } & \text { NULL } & \text { NULL } & \text { integer } \\ \text { tbot } & \text { atm_model } & \text { atm_2D_decomp1 } & \text { atm_2D_grid1 } & \text { real8 } \\ \text { qpert } & \text { atm_model } & \text { atm_2D_decomp1 } & \text { atm_3D_grid1 } & \text { real8 }\end{array}$

(b). Configuration file fields_mult_input_fields.cfg for specifying the input fields of the external algorithm.

Figure 4. Configuration files for an external algorithm.

facilitates the second approach. At each time of "configure" of a model simulation, a package of the corresponding experimental setup is automatically generated and stored. This package can be used to reproduce the existing model simulation or develop new model simulations. After creating a model simulation, users can modify the experimental setup, such as the namelist, parallel settings, hardware platform, compiling options, output settings, start and stop time, etc. After the modification of the experimental setup, users should "configure" the model simulation, and then users can "compile" and "run case". For various experiment models on various hardware platforms, users can use the same operations for various model simulations. For more information about the C-Coupler platform, please read its users' guide (Liu et al., 2014a).

The model platforms of CCSM3 and CCSM4/CESM have demonstrated that the four steps, i.e., "create case", "configure", "compile", and "run case", are sufficient and userfriendly for model simulations. We therefore used a similar four-step design for the C-Coupler platform. The most unique feature of the $\mathrm{C}$-Coupler platform is the enhancement for reproducibility of bitwise-identical simulation result for Earth system modeling. Please refer to Liu et al. (2014b) for details.

\subsection{How to couple a component model}

Generally, it takes the following steps to couple a component model with C-Coupler1:

1. Generate remapping weights if necessary.

2. Write a CoR script to register the grids of the component model and read in the remapping weights.

3. Initialize the C-Coupler runtime software system and get the MPI communicator through calling the API c_coupler_initialize, finalize the runtime software system through calling the API c_coupler_finalize, and advance the simulation time through calling the API c_coupler_advance_timer in the source code of the component model.

4. Register each parallel decomposition to C-Coupler through calling the API c_coupler_register_decomposition, register each field instance through calling the API c_coupler_register_model_data, and provide or obtain coupling fields through calling the API c_coupler_execute_procedure in the source code of the component model.

5. Write configuration files (Sect. 4.2) for the component model in order to integrate the component model into the C-Coupler platform.

We note that the steps similar to the above 1,3 , and 4 are always required when coupling a new component model with other couplers. Steps 2 and 5, which produce configuration files for coupling, are specific to C-Coupler. In C-Coupler1, these configuration files for the coupling procedures are written manually by scientists. In the future C-Coupler2, they will be generated automatically by the coupling generator.

\subsection{Enhancement for reliability of software}

Reliability is an important characteristic of software quality. To make C-Coupler1 and experiment models more reliable, more than 900 diagnoses are inserted into the source code (about 30000 lines) of C-Coupler1. These diagnoses focus on the following functions:

1. Trace the behavior of coupling during a model simulation. C-Coupler1 can trace the flow of coupling for each 


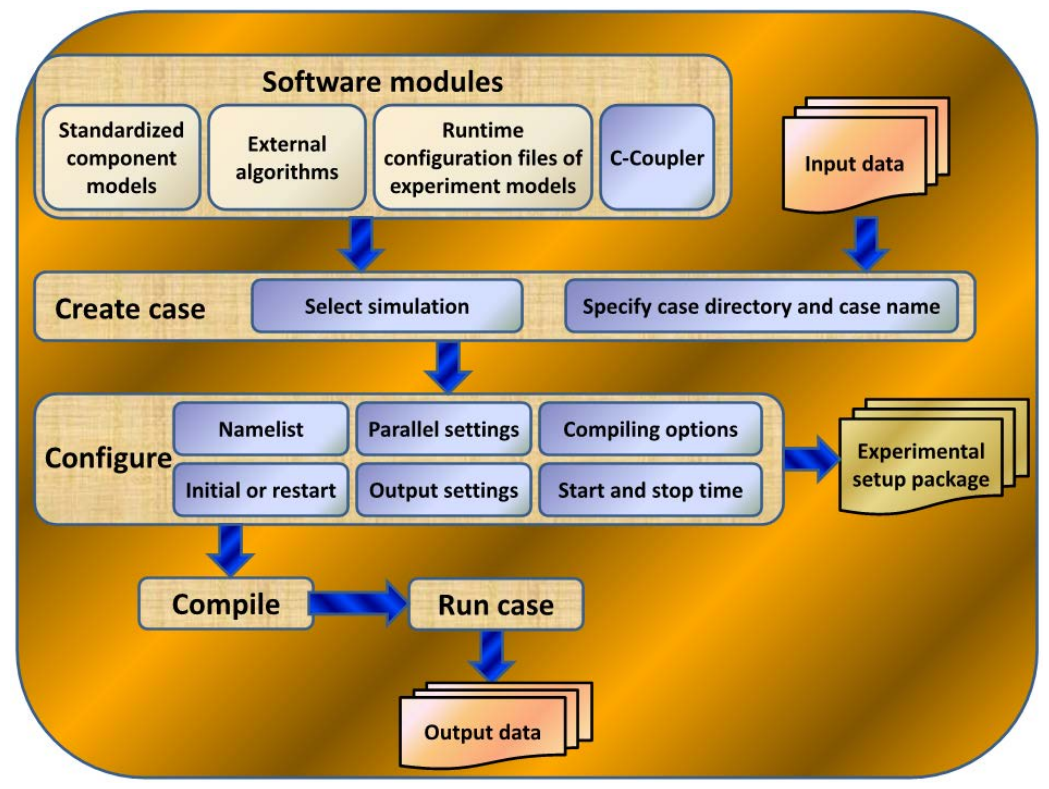

Figure 5. The general software architecture of the C-Coupler platform.

component model and the I/O fields for each runtime algorithm at each coupling step.

2. Detect errors (software bugs) in C-Coupler1. There are more than 600 diagnoses for detecting potential errors in the input and output of managers in the runtime software system of C-Coupler1.

3. Detect errors in the configuration files. C-Coupler1 can check whether a configuration file is right in format and content, and whether configuration files are consistent between component models. For example, given that a runtime algorithm transfers a number of coupling field instances from component model $\mathrm{A}$ to $\mathrm{B}$, each of $\mathrm{A}$ and $\mathrm{B}$ has a configuration file for this data transfer. CCoupler1 will check the consistency between these two configuration files.

4. Detect errors in the C-Coupler API calls. When a component model calls a C-Coupler API, C-Coupler1 will check the consistency between the API call and the corresponding configuration files. Moreover, C-Coupler1 can detect the field instances which are required as input for coupling but not provided by any component models.

In a default setting, the trace for coupling behavior is disabled because it is time-consuming and will produce a large amount of data. This detection of errors in C-Coupler1, configuration files, and C-Coupler API calls is always enabled because it only slows down the initialization for the runtime software system.

\section{Evaluation}

To evaluate C-Coupler1, we used it to construct several experiment models, including FGOALS-gc, GAMIL2-sole, GAMIL2-CLM3, MASNUM-sole, POM-sole, MASNUMPOM, and MOM4p1-sole. FGOALS-gc is a CSM version based on the CSM FGOALS-g2 (Li et al., 2013a), where the original CPL6 coupler in FGOALS-g2 is replaced by CCoupler1. GAMIL2-sole is a stand-alone component model configuration of the atmosphere model GAMIL2 (Li et al., 2013b), the atmosphere component in FGOALS-g2, which participated in the Atmosphere Model Intercomparison Project (AMIP) in CMIP5. GAMIL2-CLM3 is a coupled model configuration consisting of GAMIL2 and the land surface model CLM3 (Oleson et al., 2004). MASNUMsole is a stand-alone component model configuration of the wave model MASNUM (Yang et al., 2005). POM-sole is a stand-alone component model configuration based on a parallel version of the ocean model POM (Wang et al., 2010). MASNUM-POM is a coupled model configuration consisting of MASNUM and POM. MOM4p1-sole is a stand-alone component model version of the ocean model MOM4p1 (Griffies et al., 2010).

In the following context of this section, we will evaluate C-Coupler1 in several aspects, including the coupler component, direct coupling, 3-D coupling, code sharing, parallel performance, and the work amount for integrating a standalone component model version onto the C-Coupler platform. 


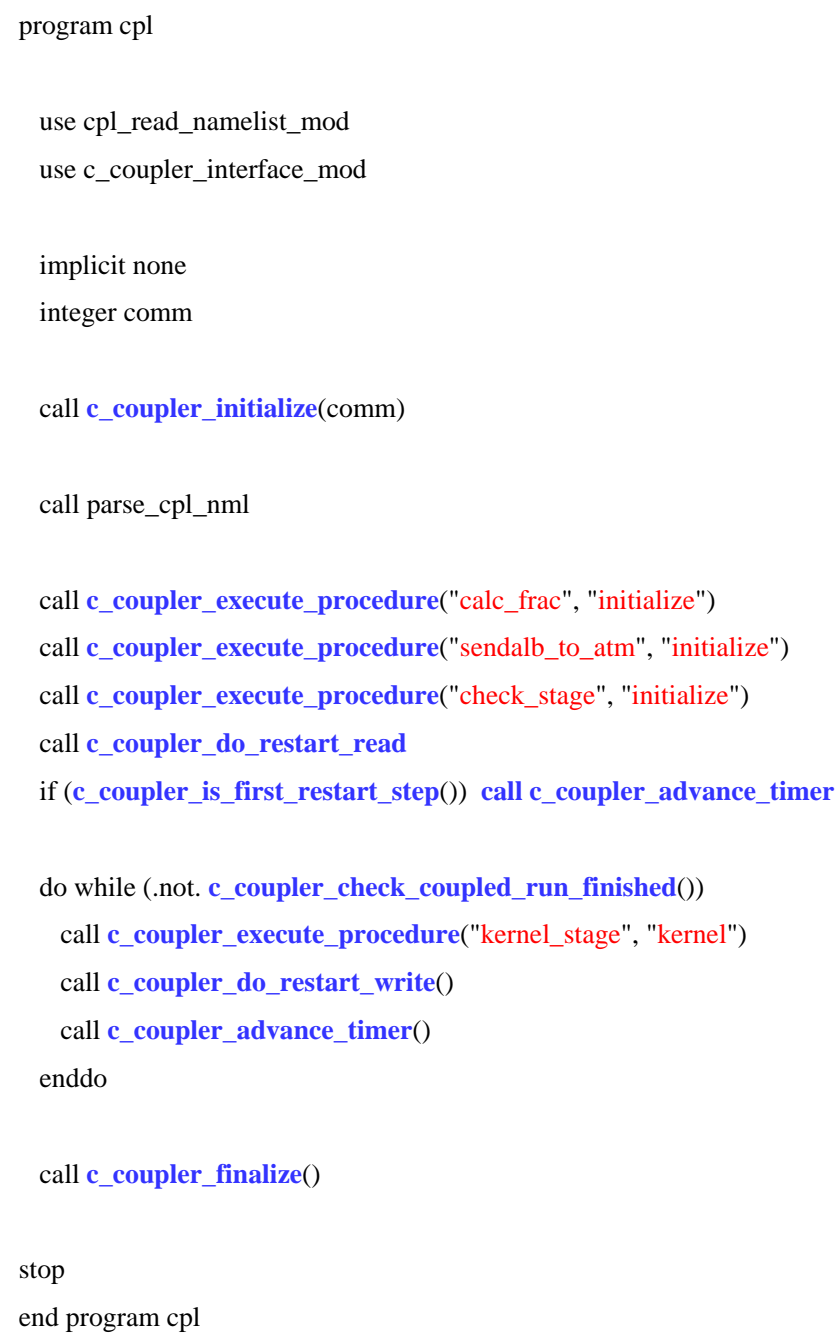

Figure 6. The code of the main driver of the coupler component in FGOALS-gc. The C-Coupler APIs are marked in blue.

\subsection{Coupler component and direct coupling}

To construct FGOALS-gc, we use C-Coupler1 to develop a separate and centralized coupler component according to the CPL6 coupler. Then the four component models in FGOALS-g2 - i.e., the atmosphere model GAMIL2, land surface model CLM3, ocean model LICOM2 (Liu et al., 2012), and an improved version of the sea ice model CICE4 (Liu, 2010) - are coupled together with the C-Coupler1 coupler component. All flux calculation algorithms in the CPL6 coupler are integrated into C-Coupler1 as external algorithms. These algorithms can be treated as public algorithms that can be shared by other experiment models. Figure 6 shows the main driver of the coupler component in FGOALS-gc. It is very simple, with a few lines of code, most of which call the C-Coupler APIs, while the main driver of the CPL6 coupler has about 1000 lines of code. This is because the coupling flow derived from the CPL6 has been

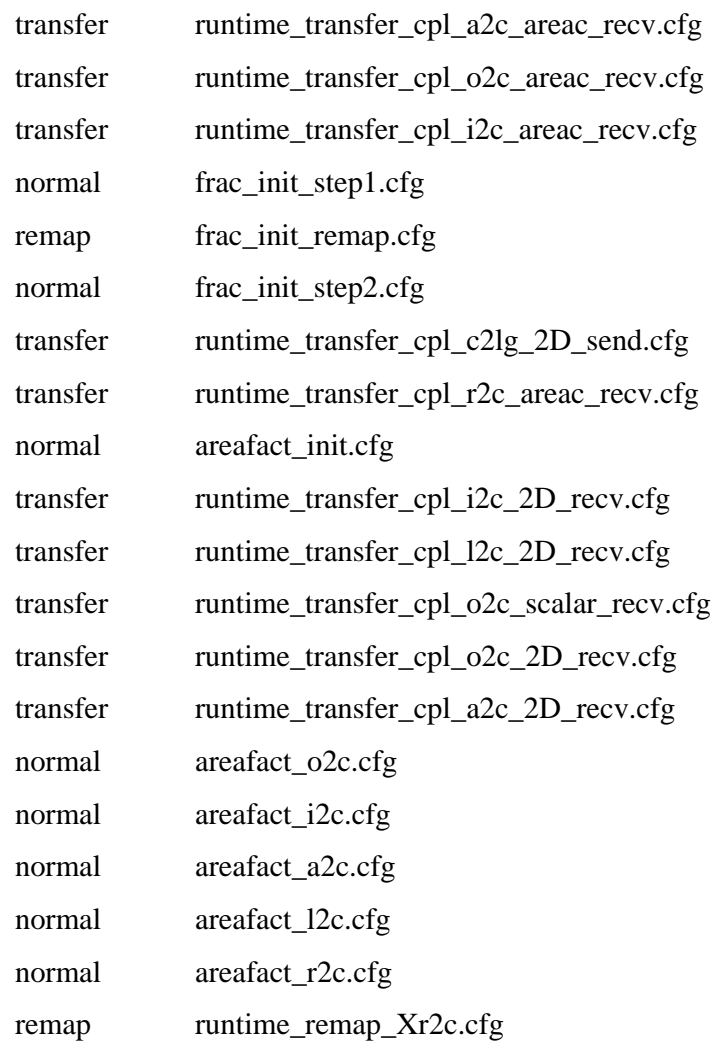

Figure 7. Part of the runtime configuration file of the algorithm list for the coupler component in FGOALS-gc. The first column specifies the type of each runtime algorithm. Transfer specifies the data transfer algorithms, remap specifies the data interpolation algorithms, and normal specifies the external algorithms. The second column specifies the configuration file of each runtime algorithm.

described by a set of configuration files that are manually defined by us. Figure 7 shows a part of the runtime configuration file of the algorithm list for the coupler component. In detail, each line is the keyword of a runtime algorithm. The first column in the keyword specifies the type of the runtime algorithm. Transfer denotes a data transfer algorithm, remap denotes a data interpolation algorithm, and normal denotes an external algorithm. The second column specifies the configuration file of the runtime algorithm. In sum, this runtime configuration file clearly lists out 91 runtime algorithms. For each runtime algorithm, there are configuration files to specify the input and output field instances.

Figure 8 shows the runtime configuration file of the runtime procedures for the coupler component, where each line corresponds to a runtime procedure. All runtime procedures share the same runtime configuration file of the algorithm list in Fig. 7. For a runtime procedure, the first column is the procedure name, which is the input parameter when model code calls the C-Coupler API c_coupler_execute_procedure. The second column and third column respectively specify the start and end index of the runtime algorithms in the algorithm 


$\begin{array}{lcc}\text { calc_frac } & 0 & 6 \\ \text { sendalb_to_atm } & 7 & 39 \\ \text { check_stage } & 40 & 44 \\ \text { kernel_stage } & 45 & 90\end{array}$

Figure 8. The runtime configuration file of the runtime procedures for the coupler component in FGOALS-gc.

list in Fig. 7. Then a runtime procedure can find the keywords of all runtime algorithms in it.

Our tests show that FGOALS-gc achieves the same (bitwise-identical) simulation result with FGOALS-g2. This result demonstrates that $\mathrm{C}$-Coupler1 can be used to construct a coupler component for a complicated coupled model, such as a CSM, without changing the simulation result. FGOALS-g2 and FGOALS-gc can be downloaded through "svn-username=guest-password=guest co http://thucpl1. 3322.org/svn/coupler/CCPL_CPL6_consistency_checking."

When constructing the experiment models GAMIL2CLM3 and MASNUM-POM, we did not build a separate coupler component but used the direct coupling where no separate executable is generated for coupling. For GAMIL2CLM3, as GAMIL2 and CLM3 share the same horizontal grid, there is only data transfer between them. For MASNUM-POM, as the grid of MASNUM is different from the grid of POM, both data transfer and data interpolation are between these two component models.

\subsection{Parallel 3-D coupling}

In MASNUM-POM, there is only one coupling field, the wave-induced mixing coefficient (Qiao et al., 2004), a 3-D field from MASNUM to POM. As the horizontal grids and vertical grids in these two component models are different, 3-D interpolation is required during coupling. In detail, we use CoR to generate the remapping weights for the 3-D interpolation. The corresponding 3-D remapping algorithm is generated through cascading two remapping algorithms: a bilinear remapping algorithm for the horizontal grids and a 1-D spline remapping algorithm for the vertical grids. For the 1D vertical interpolation, MASNUM and POM have different kinds of vertical grids: a $z$ grid for MASNUM and a sigma grid for POM.

As introduced in Sect. 5.1, MASNUM-POM uses direct coupling without a coupler component. As the resolution of MASNUM is lower than the resolution of POM, we put the calculation of the 3-D interpolation in the runtime procedure of POM in order to achieve better parallel performance. Therefore, the 3-D interpolation shares the same processes with POM. When POM runs with multiple processes, the 3$\mathrm{D}$ interpolation is computed in parallel. Our evaluation shows that the 3-D interpolation keeps the same (bitwise-identical) result with different numbers of processes.

\subsection{Code sharing}

The experiment models FGOALS-gc, GAMIL2-CLM3, and GAMIL2-sole share the same atmosphere model, GAMIL2. In FGOALS-gc, the surface fields required by GAMIL2 are provided by other component models and computed by the coupler component with C-Coupler1. In GAMIL2-CLM3, the surface fields required by GAMIL2 are provided by CLM3 and the I/O data files which contain ocean fields and sea ice fields, and computed by the private flux algorithms in GAMIL2. GAMIL2-sole is similar to GAMIL2-CLM3, while the difference is that GAMIL2-sole directly calls a land surface package to simulate the surface fields from the land. Therefore, in these three experiment models, GAMIL2 has different procedures for the surface fields.

However, we make GAMIL2 share the same code version in these three experiment models. All algorithms for computing the input surface fields in GAMIL2 have been registered to C-Coupler1 as the private external algorithms. In different experiment models, the same runtime procedures of GAMIL2 have different lists of runtime algorithms. As a result, all these experiment models keep the same (bitwiseidentical) simulation result with the original model versions without C-Coupler1.

MASNUM-POM and MASNUM-sole share the same wave model, MASNUM, while MASNUM-POM and POMsole share the same ocean model, POM. Similarly, we make MASNUM and POM share the same code in these experiment models that keeps the same (bitwise-identical) simulation result with the original model versions without CCoupler1.

\subsection{Parallel performance}

To evaluate the parallel performance of C-Coupler1, we use a high-performance computer named Tansuo100 in Tsinghua University in China. It consists of more than 700 computing nodes, each of which contains two Intel Xeon 5670 six-core CPUs and $32 \mathrm{~GB}$ main memory. All computing nodes are connected by a high-speed InfiniBand network with peak communication bandwidth $5 \mathrm{~GB} \mathrm{~s}^{-1}$. We use the Intel $\mathrm{C} / \mathrm{C}++/$ Fortran compiler version 11.1 and the Intel MPI library version 4.0 for compiling the experiment models, with optimization level $\mathrm{O} 2$ or $\mathrm{O} 3$.

This evaluation focuses on the internal algorithms implemented in the runtime software system, including the data transfer algorithm and data-remapping algorithm, without the consideration of the serial data I/O algorithm. Although the Tansuo100 computer has more than 8000 CPU cores, we can only use less than $1000 \mathrm{CPU}$ cores because a lot of other users require the use of this computer. As each computing node has 12 CPU cores, we set the number of processes (each 


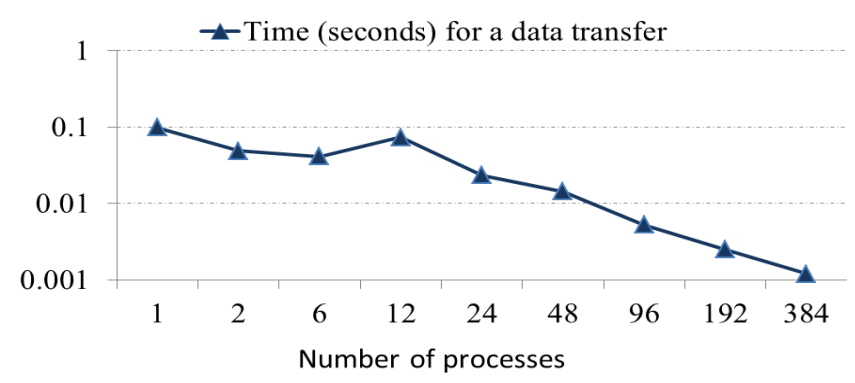

Figure 9. The scaling performance of a data transfer in MASNUMPOM: transferring the 3-D field wave-induced mixing coefficient from MASNUM to POM.

process runs on a CPU core) to an integral multiple of 12 when the number is no less than 12. A data transfer occurs between two components. When evaluating its parallel performance, the maximum number of processes for each of the corresponding two components is 384 , and the process number of the two components keeps the same in each test. The two components do not share the same computing node, and so the data transfer must go through the InfiniBand network. A data interpolation is processed within a component. When evaluating its parallel performance, the maximum number of processes for the corresponding component is 768 .

\subsubsection{Parallel performance of the data transfer algorithm}

We first use MASNUM-POM for this evaluation, where a 3-D field, the wave-induced mixing coefficient, is directly transferred from MASNUM to POM. Both the horizontal grids of MASNUM and POM have about $400000(721 \times 625)$ grid cells. For the vertical grid, MASNUM has 18 vertical levels, while POM has 30. Figure 9 shows the performance of a data transfer when gradually increasing the process number of MASNUM and POM from 1 to 384. When increasing the process number from 1 to 12 within a computing node, the performance is slightly improved. However, from process number 12 to 384 , almost linear speedup is achieved. This is because the decomposition is still coarse (more than 1000 horizontal grid cells per process, with 18 vertical levels) at 384 processes.

Next we use the data transfer from the atmospherical model GAMIL2 to the coupler component in FGOALS-gc for further evaluation, where 19 fields on the GAMIL2 horizontal grid (the grid size is $128 \times 60=7680$ ) are transferred. Figure 10 shows the performance of a data transfer. The performance is also slightly improved when increasing the process number from 1 to 12 . From process number 12 to 192, the performance is improved slowly. From process number 192 to 384, the performance gets much worse. This relatively low speedup is because, when the process number increases, the package size for MPI communication decreased, and thus

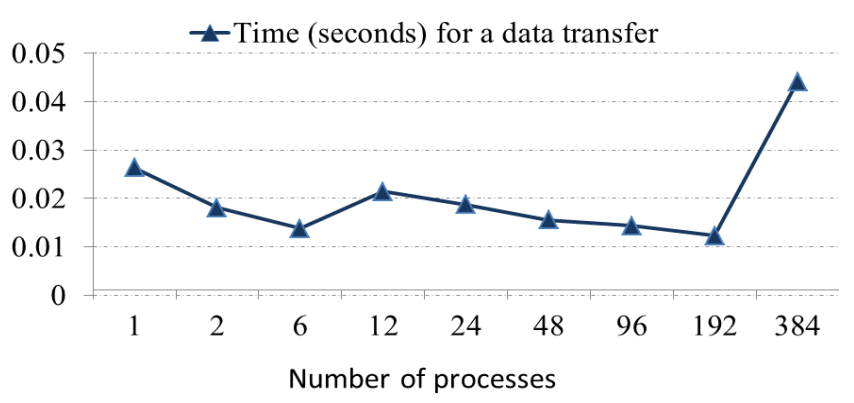

Figure 10. The scaling performance of a data transfer in FGOALSgc: transferring 19 fields on the GAMIL2 horizontal grid from GAMIL2 to the coupler component.

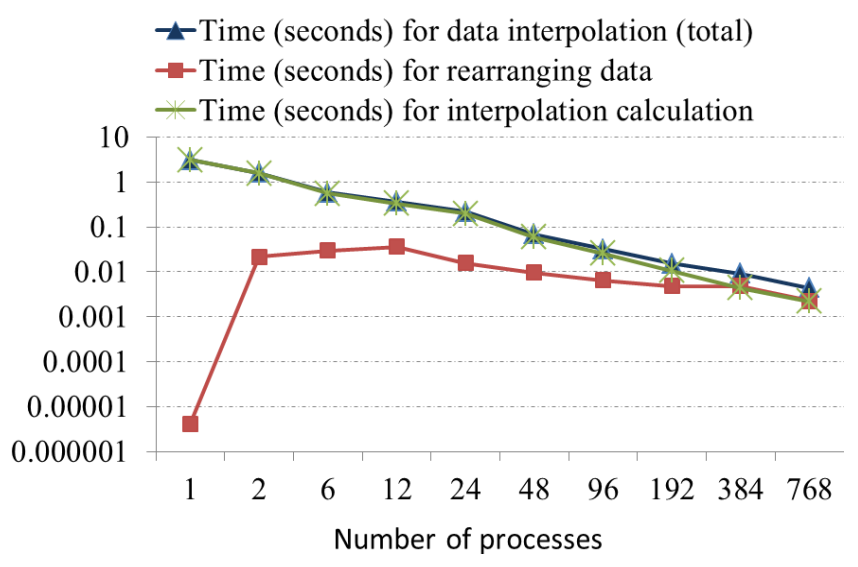

Figure 11. The scaling performance of a 3-D interpolation in MASNUM-POM: interpolating the 3-D field wave-induced mixing coefficient from the 3-D grid of MASNUM to the 3-D grid of POM on POM.

the communication bandwidth achieved in each MPI communication decreases.

\subsubsection{Parallel performance of the data-remapping algorithm}

Similarly, we first use MASNUM-POM for this evaluation, where the 3-D field, the wave-induced mixing coefficient, is interpolated from the 3-D grid of MASNUM to the 3-D grid of POM on the processes for POM. Figure 11 shows the performance of a data interpolation when gradually increasing the process number of POM from 1 to 768 , including the total time, the time for rearranging the field, and the time for interpolation calculation. For the performance of the total and interpolation calculation, almost linear speedup is achieved. However, the data rearrangement achieves much poorer performance. When increasing the process number from 12 to 768 , only 16 .x-fold speedup is achieved. This is because the package size for MPI communication decreases when increasing the process number. 


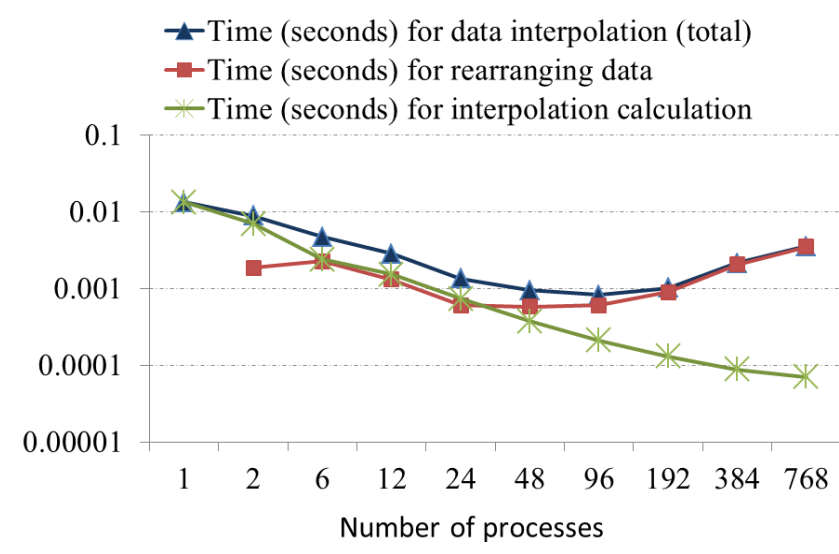

Figure 12. The scaling performance of a 2-D interpolation in FGOALS-gc: interpolating 19 horizontal fields from the GAMIL2 grid to the LICOM2 grid on the coupler component.

For further evaluation, we use the horizontal data interpolation from the atmospherical grid (GAMIL2 grid) to ocean grid (LICOM2 grid with $360 \times 196=70560$ cells) on the coupler component in FGOALS-gc, where 19 fields are interpolated. As shown in Fig. 12, the data rearrangement achieves much poorer performance speedup than the interpolation calculation. As a result, the performance speedup of the total interpolation is poor. From process number 1 to 96 , only 16-fold speedup is achieved. From processor number 96 to 768 , the total performance gets poorer. This is because the package size for MPI communication is very small when rearranging the fields.

\subsection{Integration of a stand-alone component model version}

A common model software platform can prospectively facilitate model development and scientific research through unifying the manner for operating various model simulations. As the C-Coupler platform is targeted to be a common model software platform, it should be able to flexibly integrate various models for simulations. Besides the coupled model configurations that use C-Coupler for coupling, the standalone component model versions without coupling and the coupled model versions that use other couplers but not CCoupler1 for coupling can also be integrated onto the CCoupler platform. For the corresponding evaluation, we integrated a stand-alone component model version of MOM4p1 onto the C-Coupler platform.

Section 4.4 shows the five major steps for coupling a component model with C-Coupler1. It only takes steps 3 and 5 to integrate the stand-alone MOM4p1. For step 3, less than 10 lines of source code are added to the main driver of MOM4p1. For step 5, we wrote five simple configuration files according to Sect. 4.2.1. Finally, the stand-alone MOM4p1 can be operated on the C-Coupler platform. When further coupling MOM4p1 and other component models together on the C-Coupler platform, the other steps (i.e., steps 1,2, and 4) need to be conducted.

\section{Discussion of future developments}

C-Coupler is now ready for model development with its first version C-Coupler1. As C-Coupler is a community coupler, we welcome scientists and engineers to use it and contribute to it in various aspects, such as providing component models, coupled models, flux algorithms, model simulations, bug reports, etc. Furthermore, we welcome further modeling groups in the use of C-Coupler for model development. Any new requirements from scientists, engineers, and modeling groups regarding $\mathrm{C}-\mathrm{Coupler}$ can likely be considered in the future plan for development of C-Coupler. In China, the number of users of C-Coupler is increasing, and more and more component models and coupled models are integrated on the same C-Coupler model platform.

As the first version, C-Coupler1 does not achieve all the targets of C-Coupler. For the future versions of C-Coupler, we will consider at least the following several aspects:

1. Coupling generator. The runtime software system takes the runtime configuration files of a model simulation as input. In C-Coupler1, the runtime configuration files have to be written manually by users. To facilitate the work for building a model simulation, we propose to design and develop a coupling generator in the future which can automatically generate the runtime configuration files according to the configuration files of the component models, experiment model, and external algorithms. Moreover, the coupling generator will be able to automatically optimize the parallel performance of the whole model simulation. For example, for the direct coupling between two component models with different horizontal resolutions, the coupling generator will make the corresponding data interpolation run in the runtime procedures of the component model with higher resolution in order to minimize the data size of the fields transferred between these two component models. We plan to develop the coupling generator in C-Coupler2, the second version of C-Coupler.

2. Single executable. C-Coupler1 uses multiple executables for a coupled model. A typical problem with this approach is that the processor time will be wasted when the components do not run concurrently. The CPL7 coupler has demonstrated that the approach of using a single executable with a top-level driver, which manages the processor layout and time sequencing between the components, can solve this problem and thus improve the overall parallel performance of the whole coupled model. For the future versions of C-Coupler, we will consider how to achieve a similar top-level driver in a simple way, under the general design of C-Coupler. 
For example, the top-level driver should be as simple as the main driver of the coupler component in FGOALSgc, as shown in Fig. 6.

3. Parallel performance optimization. As shown in Sect. 5.4, the parallel performance of the data transfer algorithm is not particularly good, and the dataremapping algorithm is not good enough, even poor, when the grid size is small and the parallel performance of the data transfer algorithm is not particularly good, even poor. For future work, we will try to further improve the data transfer algorithm. Moreover, we will consider how to improve the parallel performance of the whole experiment mode with C-Coupler, especially when the resolution of models increases in the future. For example, C-Coupler will provide a parallel I/O library and the coupling generator will automatically improve the overall performance when generating runtime configuration files.

4. More functions. As demonstrated in Sect. 5, CCoupler1 is able to unify various stand-alone component models and coupled models onto the same model platform. In order to unify more kinds of models onto the same model platform, the future versions of CCoupler will provide more functions to support oneway, or even two-way, model nesting, interactive coupled ensembles (Kirtman and Shukla, 2002), etc. In addition, we will consider how to make it possible for CCoupler to integrate various assimilation systems and diagnostic systems onto the same model platform.

5. More remapping algorithms. Recently, we merged the development of CoR and C-Coupler together. The available 2-D remapping algorithms in CoR are currently of low order. In the future, we will develop more 2D remapping algorithms with higher order, so as to provide more selections for model coupling and scientific researches. Moreover, we will consider developing some pure 3-D remapping algorithms in CoR, such as the 3-D $n$ neighbor distance-weighted average and trilinear remapping algorithms used in the OASIS coupler, and compare them with the "2-D + 1-D" approach.

\section{Conclusion}

C-Coupler1 is a parallel 3-D coupler which achieves bitwiseidentical simulation result with different numbers of processes. Guided by the general design of C-Coupler, CCoupler1 enables the same code of runtime software system and component models to be shared by multiple experiment models, and enables multiple experiment models to work on the same model platform. It can be used to construct a coupler component with a few lines of code for constructing a complex coupled model like CSM, and can also be used as direct coupling for better parallel performance of the whole coupled model. C-Coupler1 is ready for developing CSMs and ESMs. In China, C-Coupler is beginning to assist more and more in model development, e.g., the development of a CSM at Tsinghua University targeting CMIP6; the development of the atmosphere model GAMIL at the State Key Laboratory of Numerical Modeling for Atmospheric Sciences and Geophysical Fluid Dynamics (LASG), Institute of Atmospheric Physics; and the development of the coupled model MASNUM-POM at the first Institute of Oceanography, State Oceanic Administration. We have begun development of a uniform model platform which will integrate various component models, experiment models, and model simulations together. We believe that C-Coupler will help advance Earth system modeling.

\section{Code availability}

The source code of CoR can be downloaded through "svn-username=guest-password=guest co http://thucpl1.3322.org/svn/coupler/CoR1.0". The source code of C-Coupler1 can be downloaded with FGOALSgc through "svn-username=guest - password=guest co http://thucpl1.3322.org/svn/coupler/CCPL_CPL6_ consistency_checking.". If any problems are encountered with the code, please feel free to contact us (liulicess@ tsinghua.edu.cn).

\section{The Supplement related to this article is available online at doi:10.5194/gmd-7-2281-2014-supplement.}

Acknowledgements. This work is supported in part by the Natural Science Foundation of China (no. 41275098), the National Grand Fundamental Research 973 Program of China (no. 2013CB956603), and the Tsinghua University Initiative Scientific Research Program (no. 20131089356).

Edited by: S. Easterbrook

\section{References}

Armstrong, C. W., Ford, R. W., and Riley, G. D.: Coupling integrated Earth System Model components with BFG2, Concurr. Comp.-Pract. E, 21, 767-791, doi:10.1002/cpe.1348, 2009.

Balaji, V., Anderson, J., Held, I., Winton, M., Durachta, J., Malyshev, S., and Stouffer, R. J.: The Exchange Grid: a mechanism for data exchange between Earth System components on independent grids, in: Proceedings of the 2005 International Conference on Parallel Computational Fluid Dynamics, College Park, MD, USA, Elsevier, 2006.

Bao, Q., Lin, P., Zhou, T., Liu, Y., Yu, Y., Wu, G., He, B., He, J., Li, L., Li, J., Li, Y., Liu, H., Qiao, F., Song, Z., Wang, B., 
Wang, J., Wang, P., Wang, X., Wang, Z., Wu, B., Wu, T., Xu, Y., Yu, H., Zhao, W., Zheng, W., and Zhou, L.: The Flexible Global Ocean-Atmosphere-Land System Model, spectral version 2: FGOALS-s2, Adv. Atmos. Sci., 30, 561-576, 2013.

Collins, W. D., Bitz, C. M., Blackmon, M. L., Bonan, G. B., Bretherton, C. S., Carton, J. A., Chang, P., Doney, S. C., Hack, J. J., Henderson, T. B., Kiehl, J. T., Large, W. G., McKenna, D. S., Santer, B. D., and Smith, R. D.: The Community Climate System Model Version 3 (CCSM3), J. Climate, 19, 2122-2143, 2006.

Craig, A. P., Jacob, R. L., Kauffman, B., Bettge, T., Larson, J. W., Ong, E. T., Ding, C. H. Q., and He, Y.: CPL6: the new extensible, high performance parallel coupler for the Community Climate System Model. Int. J. High Perform. C., 19, 309-327, 2005.

Craig, A. P., Vertenstein, M., and Jacob, R.: A new flexible coupler for Earth System Modelling developed for CCSM4 and CESM1, Int. J. High Perform. C, 26, 31-42, doi:10.1177/1094342011428141, 2012.

Donner, L. J., Wyman, B. L., Hemler, R. S., Horowitz, L. W., Ming, Y., Zhao, M., Golaz, J. C., Ginoux, P., Lin, S. J., Schwarzkopf, M. D., Austin, J., Alaka, G., Cooke, W. F., Delworth, T. L., Freidenreich, S. M., Gordon, C. T., Griffies, S. M., Held, I. M., Hurlin, W. J., Klein, S. A., Knutson, T. R., Langenhorst, A. R., Lee, H.-C., Lin, Y., Magi, B. I., Malyshev, S. L., Milly, P. C. D., Naik, V., Nath, M. J., Pincus, R., Ploshay, J. J., Ramaswamy, V., Seman, C. J., Shevliakova, E., Sirutis, J. J., Stern, W. F., Stouffer, R. J., Wilson, R. J., Winton, M., Wittenberg, A. T., and Zeng, F.: The dynamical core, physical parameterizations, and basic simulation characteristics of the atmospheric component AM3 of the GFDL Global Coupled Model CM3, J. Climate, 24, 3484-3519, 2011.

Dufresne, J.-L., Foujols, M.-A., Denvil, S., Caubel, A., Marti, O., Aumont, O., Balkanski, Y., Bekki, S., Bellenger, H., Benshila, R., Bony, S., Bopp, L., Braconnot, P., Brockmann, P., Cadule, P., Cheruy, F., Codron, F., Cozic, A., Cugnet, D., de Noblet, N., Duvel, J.-P., Eth'e, C., Fairhead, L., Fichefet, T., Flavoni, S., Friedlingstein, P., Grandpeix, J.-Y., Guez, L., Guilyardi, E., Hauglustaine, D., Hourdin, F., Idelkadi, A., Ghattas, J., Joussaume, S., Kageyama, M., Krinner, G., Labetoulle, S., Lahellec, A., Lefebvre, M- P., Lefevre, F., Levy, C., Li, Z. X., Lloyd, J., Lott, F., Madec, G., Mancip, M., Marchand, M., Masson, S., Meurdesoif, Y., Mignot, J., Musat, I., Parouty, S., Polcher, J., Rio, C., Schulz, M., Swingedouw, D., Szopa, S., Talandier, C., Terray, P., and Viovy, N.: Climate change projections using the IPSL-CM5 Earth System Model: from CMIP3 to CMIP5, Clim. Dynam., 40, 2123-2165, 2013.

Dunne, J. P., John, J. G., Adcroft, A. J., Griffies, S. M., Hallberg, R. W., Shevliakova, E. N., Stouffer, R. J., Cooke, W., Dunne, K. A., Harrison, M. J., Krasting, J. P., Levy, H., Malyshev, S. L., Milly, P. C. D., Phillipps, P. J., Sentman, L. T., Samuels, B. L., Spelman, M. J., Winton, M., Wittenberg, A. T., and Zadeh, N.: GFDL's ESM2 global coupled climate-carbon Earth System Models, Part I: Physical formulation and baseline simulation characteristics, J. Climate, 25, 6646-6665, doi:10.1175/JCLI-D-11-00560.1, 2012.

Ford, R. W., Riley, G. D., Bane, M. K., Armstrong, C. W., and Freeman, T. L.: GCF: a general coupling framework, Concurr. Comp.-Pract. E, 18, 163-181, 2006.
Gent, P. R., Danabasoglu, G., Donner, L. J., Holland, M. M., Hunke, E. C., Jayne, S. R., Lawrence, D. M., Neale, R. B., Rasch, P. J., Vertenstein, M., Worley, P. H., Yang, Z.-L., and Zhang, M.: The Community Climate System Model version 4, J. Climate, 24, 4973-4991, 2011.

Giorgetta, M. A., Jungclaus, J. H., Reick, C. H., Legutke, S., Bader, J., Böttinger, M., Brovkin, V., Crueger, T., Esch, M., Fieg, K., Glushak, K., Gayler, V., Haak, H., Hollweg, H.-D., Ilyina, T., Kinne, S., Kornblueh, L., Matei, D., Mauritsen, T., Mikolajewicz, U., Mikolajewicz, U., Mueller, W., Notz, D., Pithan, F., Raddatz, T., Rast, S., Redler, R., Roeckner, E., Schmidt, H., Schnur, R., Segschneider, J., Six, K. D., Stockhause, M., Timmreck, C., Wegner, J., Widmann, H., Wieners, K.-H., Claussen, M., Marotzke, J., and Stevens, B.: Climate and carbon cycle changes from 1850 to 2100 in MPI-ESM simulations for the Coupled Model Intercomparison Project phase 5, J. Adv. Model. Earth Syst., 5, 572-597, 2013.

Griffies, S. M., Schmidt, M., and Herzfeld, M.: Elements of MOM4p1, GFDL Ocean Group Technical Report 6, Geophysical Fluid Dynamics Laboratory, Princeton, 2010.

Hazeleger, W., Wang, X., Severijns, C., Stefanescu, S., Bintanja, R., Sterl, A., Wyser, K., Semmler, T., Yang, S., van den Hurk, B., van Noije, T., van der Linden, E., and van der Wiel, K.: EC-Earth V2.2: description and validation of a new seamless earth system prediction model, Clim. Dynam., 39, 2611-2629, doi:10.1007/s00382-011-1228-5, 2011.

Hill, C., DeLuca, C., Balaji, V., Suarez, M., and da Silva, A.: Architecture of the Earth System Modelling Framework, Comput. Sci. Eng., 6, 18-28, 2004.

Hurrell, J. W., Holland, M. M., Gent, P. R., Ghan, S., Kay, J. E., Kushner, P. J., Lamarque, J.-F., Large, W. G., Lawrence, D., Lindsay, K., Lipscomb, W. H., Long, M. C., Mahowald, N., Marsh, D. R., Neale, R. B., Rasch, P., Vavrus, S., Vertenstein, M., Bader, D., Collins, W. D., Hack, J. J., Kiehl, J., and Marshall, S.: The Community Earth System Model: a framework for collaborative research, B. Am. Meteorol. Soc., 94, 1339-1360, 2013.

Jacob, R., Larson, J., and Ong, E.: $M \times N$ Communication and Parallel Interpolation in Community Climate System Model version 3 using the Model Coupling Toolkit, Int. J. High Perform. C, 19, 293-307, 2005.

Ji, D., Wang, L., Feng, J., Wu, Q., Cheng, H., Zhang, Q., Yang, J., Dong, W., Dai, Y., Gong, D., Zhang, R.-H., Wang, X., Liu, J., Moore, J. C., Chen, D., and Zhou, M.: Description and basic evaluation of Beijing Normal University Earth System Model (BNU-ESM) version 1, Geosci. Model Dev., 7, 2039-2064, doi:10.5194/gmd-7-2039-2014, 2014.

Jones, P.: First and second-order conservative remapping schemes for grids in spherical coordinates, Mon. Weather Rev., 127, 2204-2210, 1999.

Jungclaus, J. H., Fischer, N., Haak, H., Lohmann, K., Marotzke, J., Matei, D., Mikolajewicz, U., Notz, D., and von Storch, J. S.: Characteristics of the ocean simulations in MPIOM, the ocean component of the MPI-Earth System Model, J. Adv. Model. Earth Syst., 5, 422-446, 2013.

Kirtman, B. P. and Shukla, J.: Interactive coupled ensemble: a new coupling strategy for CGCMs, Geophys. Res. Lett., 29, 5-1-5-4, 2002. 
Larson, J., Jacob, R., and Ong, E.: The Model Coupling Toolkit: a new Fortran90 Toolkit for building multiphysics parallel coupled models, Int. J. High Perform, C, 19, 277-292, 2005.

Li, L. J., Lin, P. F., Yu, Y. Q., Wang, B., Zhou, T. J., Liu, L., Liu, J. P., Bao, Q., Xu, S. M., Huang, W. Y., Xia, K., Pu, Y., Dong, L., Shen, S., Liu, Y. M., Hu, N., Liu, M. M., Sun, W. Q., Shi, X. J., Zheng, W. P., Wu, B., Song, M.-R., Liu, H. L., Zhang, X. H., Wu, G. X., Xue, W., Huang, X. M., Yang, G. W., Song, Z. Y., and Qiao, F. L.: The Flexible Global Ocean-Atmosphere-Land System Model: grid-point version 2: FGOALS-g2, Adv. Atmos. Sci., 30, 543-560, 2013a.

Li, L. J., Wang, B., Dong, L., Liu, L., Shen, S., Hu, N., Sun, W., Wang, Y., Huang, W., Shi, X., Pu, Y., G. and Yang.: Evaluation of Grid-point Atmospheric Model of IAP LASG version 2 (GAMIL2), Adv. Atmos. Sci., 30, 855-867, doi:10.1007/s00376013-2157-5, 2013b.

Liu, H. L., Lin, P. F., Yu, Y. Q., and Zhang, X. H.: The baseline evaluation of LASG/IAP Climate system Ocean Model (LICOM) version 2.0, Acta Meteorol. Sin., 26, 318-329, 2012.

Liu, J.: Sensitivity of sea ice and ocean simulations to sea ice salinity in a coupled global climate model, Sci. China Ser. D, 53, 911916, 2010.

Liu, L., Yang, G., and Wang, B.: CoR: a multi-dimensional common remapping software for Earth System Models, in: The Second Workshop on Coupling Technologies for Earth System Models (CW2013), available at: https://wiki.cc.gatech.edu/CW2013/ index.php/Program (last access: 8 May 2014), 2013a.

Liu, L., Yang, G., and Wang, B.: Common Multidimensional Remapping Software CoR (Common Remap) V1.0 User Reference Manual, available at: http://www.cess.tsinghua.edu.cn/publish/ess/7687/ 20120515135108504806682/Common Multi-dimensional Remapping Software CoR(Common Remap) V1.0.pdf (last access: 8 May 2014), 2013b.

Liu, L., Li, R., Zhang, C., Yang, G., and Wang, B.: Community coupler C-Coupler1 User's Guide, available at: http://www.cess. tsinghua.edu.cn/publish/ess/7687/20120515135108504806682/ CommunityCouplerC-Coupler1UserGuide.pdf (last access: 8 May 2014), 2014a.

Liu, L., Li, R., Zhang, C., Yang, G., and Wang, B.: Enhancing reproducibility of numerical simulation result on the CCoupler platform, Geosci. Model Dev. Discuss., 7, 4429-4461, doi:10.5194/gmdd-7-4429-2014, 2014b.

Oleson, K. W., Dai, Y., Bonan, G. B.,Bosilovichm, M., Dickinson, R., Dirmeyer, P., Hoffman, F., Houser, P., Levis, S., Niu, G.-Y., Thornton, P., , Vertenstein, Yang, Z., and Zeng, X.: Technical Description of the Community Land Model (CLM), NTIS \#PB2004-105836, 2004.
Qiao, F., Yuan, Y., Yang, Y., Zheng, Q., Xia, C., and Ma, J.: Waveinduced mixing in the upper ocean: distribution and application in a global ocean circulation model, Geophys. Res. Lett., 31, L11303, doi:10.1029/2004GL019824, 2004.

Redler, R., Valcke, S., and Ritzdorf, H.: OASIS4 - a coupling software for next generation Earth System Modelling, Geosci. Model Dev., 3, 87-104, doi:10.5194/gmd-3-87-2010, 2010.

Valcke, S.: The OASIS3 coupler: a European climate modelling community software, Geosci. Model Dev., 6, 373-388, doi:10.5194/gmd-6-373-2013, 2013a.

Valcke, S.: OASIS3-MCT, a coupler for climate modelling, in: The Second Workshop on Coupling Technologies for Earth System Models (CW2013), available at: https://wiki.cc.gatech.edu/ CW2013/index.php/Program (last access: 8 May 2014), 2013b.

Valcke, S., Balaji, V., Craig, A., DeLuca, C., Dunlap, R., Ford, R. W., Jacob, R., Larson, J., O'Kuinghttons, R., Riley, G. D., and Vertenstein, M.: Coupling technologies for Earth System Modelling, Geosci. Model Dev., 5, 1589-1596, doi:10.5194/gmd-5-1589-2012, 2012a.

Valcke, S., Craig, T., and Coquart, L.: OASIS3-MCT User Guide, OASIS3-MCT 1.0, CERFACS Technical Report, TR/CMGC/12/49, Toulouse, France, available at: http://pantar.cerfacs.fr/globc/publication/technicalreport/2012/ oasis3mct_UserGuide.pdf (last access: 8 May 2014), 46 pp., 2012 b.

Vichi, M., Manzini, E., Fogli, P. G., Alessandri, A., Patara, L., Scoccimarro, E., Masina, S., and Navarra, A.: Global and regional ocean carbon uptake and climate change: sensitivity to a substantial mitigation scenario, Clim. Dynam., 37, 1929-1947, doi:10.1007/s00382-011-1079-0, 2011.

Voldoire, A., Sanchez-Gomez, E., Salas y M'elia, D., Decharme, B., Cassou, C., S'en'esi, S., Valcke, S., Beau, I., Alias, A., Chevallier, M., D'equ'e, M., Deshayes, J., Douville, H., Fernandez, E., Madec, G., Maisonnave, E., Moine, M.-P., Planton, S., SaintMartin, D., Szopa, S., Tyteca, S., Alkama, R., Belamari, S., Braun, A., Coquart, L., and Chauvin, F.: The CNRM-CM5.1 global climate model: description and basic evaluation, Clim. Dynam., 40, 2091-2121, 2013.

Wang, G., Qiao, F., and Xia, C.: Parallelization of a coupled wavecirculation model and its application, Ocean Dynam., 60, 331339, 2010.

Yang, Y., Qiao, F., Zhao, W., Y. Teng and Yuan, Y.: MASNUM ocean wave numerical model in spherical coordinates and its application, Acta Oceanol. Sin., 27, 1-7, 2005. 